\title{
2nd EFLM-BD European Conference on Preanalytical Phase Preanalytical quality improvement - in quality we trust
}

\author{
Hotel Westin, Zagreb, Croatia \\ March 1-2, 2013
}

\section{Organizers:}

European Federation of Clinical Chemistry and Laboratory Medicine (EFLM)

in collaboration with:

BD Diagnostics, Preanalytical Systems

\section{Organizing Committee:}

Ana-Maria Simundic (chair), Croatia

Silvia Cattaneo, Italy

Marijana Miler, Croatia

Lora Dukic, Croatia

Sanja Dobrijevic, Croatia

\section{Scientific Committee:}

Ana-Maria Simundic (chair), Croatia

Michael Cornes, United Kingdom

Kjell Grankvist, Sweden

Giuseppe Lippi, Italy

Mads Nybo, Denmark

Svjetlana Kovalevskaya, Russia

Ludek Sprongl, Czech

Zorica Sumarac, Serbia

Stephen Church, United Kingdom 


\section{P1}

\section{Experimental study of blood tubes underfilling for routine coagulation testing}

\author{
Lippi G*1, Salvagno GL2 , Lima-Oliveira G², Funk-Adcock DM³, \\ Guidi GC2 ${ }^{2}$ Favaloro EJ4 \\ 'Laboratory of Clinical Chemistry and Hematology, Department \\ of Pathology and Laboratory Medicine, Academic Hospital of \\ Parma, Italy \\ 2Section of Clinical Biochemistry, Department of Life and \\ Reproductive Sciences, University of Verona, Italy \\ ${ }^{3}$ Esoterix, Inc., Englewood Colorado, USA \\ ${ }^{4}$ Department of Haematology, Institute of Clinical Pathology \\ and Medical Research (ICPMR), Westmead Hospital, NSW, \\ Australia
}

Background: The preanalytical phase represents the major source of variability in laboratory diagnostics. Our aim was to assess to what extent underfilling of primary blood tubes may impact upon routine coagulation testing.

Materials and methods: Blood was drawn by syringe from 21 healthy volunteers and 6 patients on warfarin therapy, and immediately transferred into $3.6 \mathrm{~mL}$ vacuum tubes containing $3.2 \%$ sodium citrate (Terumo Europe N.V., Leuven, Belgium). All tubes were filled using standardized volumes of whole blood to produce scalar amounts of filling: $3.6 \mathrm{~mL}$ (i.e., 100\%), $3.2 \mathrm{~mL}(89 \%), 2.8 \mathrm{~mL}(78 \%)$ and $2.4 \mathrm{~mL}(67 \%)$. Samples were mixed and centrifuged at $1300 \times \mathrm{g}$ for $10 \mathrm{~min}$. The plasma was tested for prothrombin time (PT), activated partial thromboplastin time (APTT) and fibrinogen (FBG) on $\mathrm{ACL}$ TOP (Instrumentation Laboratory - IL, Milan, Italy), using IL reagents. A polynomial plot was derived for each parameter from interpolation of clotting values obtained with different percentages of filling, and these plots were compared with quality specifications ( \pm 2.0 for PT, \pm 2.3 for APTT and \pm 4.8 for FBG) to calculate the minimal filling volume required to produce clinically acceptable results.

Results: The equations were (PF, Percentage of filling): PT (sec) = 3.375 x PF^2-6.255 x PF + $17.806(r=$ 0.980); $\mathrm{APTT}(\mathrm{sec})=8.925 \times \mathrm{PF} \wedge 2-23.578 \times \mathrm{PF}+$ $46.356(r=0.979) ;$ and FBG $(\mathrm{mg} / \mathrm{dL})=-311.5 \times \mathrm{PF} \wedge 2$
$+422.1 \times \mathrm{PF}+147.07(r=0.994)$. According to these equations, the minimum allowed thresholds of blood tubes filling were $>61 \%$ for PT, $>87 \%$ for APTT and $>71 \%$ for FBG.

Conclusions: Our results confirm that routine coagulation testing performed on underfilled tubes may generate biased and clinically misleading test results. This is particularly critical for APTT, wherein tubes filled at less than $\sim 90 \%$ generate unreliable data. The FBG and the PT seem more resistant to underfilling, clinical significant biases being observed only where blood tubes were filled at less than $\sim 60$ and $\sim 70 \%$, respectively.

Key words: preanalytical variability; blood tubes; coagulation testing; filling; anticoagulant

*Corresponding author: glippi@ao.pr.it

\section{P3}

\section{Determination of fibrinogen in lipemic plasma samples using optical measurement technique}

\section{Radišić Biljak V*, Božičević S, Vučić Lovrenčić M}

Merkur Teaching Hospital, Institute of Clinical Chemistry and Laboratory Medicine, Zagreb, Croatia

Background: Coagulation assays performed with photo-optical coagulometers may be affected by interfering substances, including lipids. According to the NCCLS guideline clotting parameters in lipemic specimens should ideally be measured by mechanical or electromechanical methods, which are not always available. Elimination of interference by ultracentrifugation of lipemic plasma samples may affect accuracy due to concomitant sedimentation of fibrinogen. We aimed to determine the accuracy of fibrinogen measurement in lipemic plasma samples cleared by high-speed centrifugation using an optical measurement procedure.

Materials and methods: Twenty-two lipemic plasma samples with triglyceride concentration range of 6.01 to $40.18 \mathrm{mmol} / \mathrm{L}$ were used in this study. Concentration of fibrinogen was measured 
using modified Clauss method by two measuring principles: 1) opto-mechanical measurement, accurate for lipemic samples on Siemens BFT ${ }^{\mathrm{TM}}$ II Analyzer using Siemens Multifibren* $U$ reagent in native lipemic plasma samples; and 2) optical measurement on Siemens Sysmex CA-560 System using Dade Thrombin Reagent in plasma aliquots cleared of lipemia by high-speed centrifugation (10500 x g, 15 minutes). The results were compared using Passing \& Bablok regression. Interference of triglycerides on bias was checked by linear regression. All results were analyzed using MedCalc 9.4.2.0 statistical software (MedCalc Software bvba, Mariakerke, Belgium).

Results: Passing \& Bablok regression revealed no statistically significant difference between the two compared procedures (regression equation: $y=$ $-0.6447+1.2105 x$; Intercept $\mathrm{A}=-0.6447,95 \% \mathrm{Cl}=$ -1.1500 to 0.0000 ; Slope $B=1.2105,95 \% \mathrm{Cl}=1.0000$ to 1.4000). Cusum test for linearity showed no significant deviation from linearity $(P>0.10)$. Bias between the two methods (expressed as percentage, $\%)$ was unaffected by varying triglyceride levels $\left(R^{2}\right.$ $=0.03030, P=0.438$ ).

Conclusions: Plasma fibrinogen can be accurately measured using a photo-optical system in grossly lipemic plasma samples cleared of lipemia by highspeed centrifugation.

Key words: fibrinogen; lipid interference; highspeed centrifugation; optical method

*Corresponding author: Vanja.Radisic.Biljak@idb.hr

\section{P4}

\section{Evaluation of outpatients' satisfaction questionnaire}

\author{
Milinković N*, Šumarac Z, Ignjatović S, Majkić-Singh N
}

Institute of Medical Biochemistry, Clinical Center of Serbia, Belgrade, Serbia

Background: Self-report questionnaire is the most widely used method to assess patients' satisfaction with medical care. Outpatients' satisfaction is a key aspect of the evaluation of the quality of laboratory services in tertiary health institutions. The aim of this study is to determine the relationship between outpatients' satisfaction with their laboratory service.

Materials and methods: We examined 778 outpatients using a questionnaire, indicating the essential aspects of laboratory services. Three dimensions of outpatients' satisfaction were identified: professional care, the organization of laboratory reception and perceived time spent during a laboratory visit.

Results: There was no significant difference in the gender of patients, but there were significantly ( $r=$ $0.165, \mathrm{P}<0.01$ ) more patients that were using laboratory services more than 3 years, compared to the first visit to the laboratory. In addition, $45.6 \%$ of outpatients were using laboratory service more than 3 years, while $62.4 \%$ of outpatients were older than 50 years of life. We found a statistically significant correlation $(r=0.280, P<0.01)$ between the quality of services provided and technicians' behaviour toward patients. The main dissatisfaction was waiting for half an hour in a line for blood sampling. However, $90 \%$ of outpatients wrote positive remarks for the analyzed dimensions of patients satisfaction $(P<0.05)$.

Conclusions: Estimation of outpatients' questionnaires is essential in assessing the quality of the treatment of patients, in laboratory services in tertiary health institutions. This could help optimize laboratory services and also standardize the preanalytical phase.

Key words: laboratory; outpatients; quality indicators; questionnaire

*Corresponding author: nedanvkvc@yahoo.com 


\section{P5}

\section{Free beta human chorionic gonadotropin and pregnancy-associated plasma protein A in the first trimester screening.}

\author{
Trujillo Arribas E*, Camacho Martinez P, García Pinilla M, \\ Macher Manzano H, Guerrero Montávez JM \\ Hospital Universitario Virgen del Rocio, Sevilla, Spain
}

Background: The aim of this research is to study the influence of the lapsed time between the extraction and the determination procedure in the case of free beta human chorionic gonadotropin (free beta hCG) and pregnancy-associated plasma protein A (PAPP-A), and its effect on the calculation of risk of chromosomal abnormalities. Free beta hCG and PAPP-A increases over time, but it is to be determined how long it needs to cause a significant difference on the values and on the associated risk for the prenatal screening.

Materials and methods: A group of 38 women in the first trimester of pregnancy were included in the study, blood samples were taken during the routine check-up. Blood samples were stored and transported to Virgen del Rocío Laboratories in the same conditions (uncentrifuged and in isothermal iceboxes). Once in the laboratory the samples were centrifuged and analyzed using the electrochemiluminescence immunoassay "ECLIA" on cobas 8000 (Roche Diagnostics).

Results: Both beta hCG and PAPPA increase over time. There were significant changes among the determinations corresponding to the samples extracted four, seven, and nine hours before the analysis ( $P=0.007)$. Both beta hCG and PAPPA have a linear ascending trend as the statistics and the profile graphs indicate $(P<0.001)$. Comparing two to two (the different levels of the factor -time elapsed since the extraction-), the critical levels associated to every comparison show that there are relevant differences among all the combinations of the different levels for both variables $(P<0.001)$. The associated risk has notable difference among the determinations carried out at different times from the extraction $(P<0.001)$. Such a difference, however, has no influence on the screening results.

Conclusions: Normal handling of samples is likely to increase the results of the determination of free beta hCG y PAPP-A as time till their processing increases. However, in most cases, these variations do not affect the screening results (positive or negative screening).

Key words: preanalytical phase; free beta human chorionic gonadotropin; pregnancy-associated plasma protein $\mathrm{A}$; first trimester screening

*Corresponding author: elenatrujilloarribas@gmail.com

\section{P6}

\section{Implementation of UNE-EN ISO 15189 in Catlab's pre-analytical area}

\author{
Barba N*, Alsius A, Caballé I, Navajas F, Camacho V, Crespo T
}

The SEQC (Spanish Society of Clinical Chemistry) Committee for the Extra-analytical Quality Assessment

Introduction and objectives: Catlab began operating in June 2008, in June 2010 the UNE-EN ISO 9001:2088 was obtained, one of the objectives of Quality for 2012 was to obtain the UNE-EN ISO 15189 in the pre-analytical area and in the automated serum and urine biochemistry magnitudes.

Material and methods: Catlab covers 850,000 people, with 3,300 daily requests and 7.5 million yearly measurements it services three hospitals and 96 primary care centres. The scope of the preanalytical area audit was "blood sampling and management and handling of blood and urine samples". Since the management of the 96 centers does not depend on Catlab, all processes had to be standardized. Working groups were formed with specialized staff and working protocols in procurement centres were drafted as well as standard operating procedures such as: "Venous and arterial blood extraction" or "Conditions of arrival of the samples at Catlab". The catalog, sampling instructions and procedures are updated and available from the website (www.catlab.cat). The T\&T 
system (BD) was established to monitor time and temperature during samples transport in each route. Courier arrivals and departures and the time lapse between arrival of the refrigerators until opened is monitored by an internal software. Preanalytical incidences are reported daily and, monthly, a progress summary is submitted to centers. Joint sessions are conducted with nurses and procurement centres clinicians. Catlab participates in two external quality programs: SEQC and CAP. Eight indicators of preanalytical quality have been defined.

Results and conclusions: On the $11^{\text {th }}$ of May 2012, Catlab's pre-analytical area was granted the UNEEN 15189 accreditation. This involves standardization and improvement of a little automated processes. Continuous improvement is pursued.

Key words: UNE-EN ISO 15189; pre-analitycal area

*Corresponding author: nbarba@catlab.cat

\section{P7}

\section{Influence of type of urine sample on use of albumin as renal biomarker}

\author{
Dajak $M^{* 1}$, Ignjatović $S^{2}$, Stojimirović $B^{3}$, Majkić-Singh $N^{2}$ \\ ${ }^{1}$ Center of Medical Biochemistry, Clinical Center of Serbia, \\ Belgrade, Serbia \\ ${ }^{2}$ Center of Medical Biochemistry, Clinical Center of Serbia and \\ School of Pharmacy, Belgrade, Serbia \\ ${ }^{3}$ Center of Urology and Nephrology, Clinical Center of Serbia \\ and School of Medicine, Belgrade, Serbia
}

Background: Albuminuria is one of the most significant biomarkers of early detection, management, prognosis and progression of kidney disease. In clinical practice it has not yet been clarified which urine sample is most appropriate for albuminuria measurement. The difficulty of collecting 24-h urine samples has led to surrogate measurements of albumin excretion rate. Some authors consider that corrections with urinary creatinine can be performed only in primary glomerulopathy with no tubular damage. The aim of this study was to compare urinary albumin values in two different urine samples.
Materials and methods: The study included 117 patients with chronic kidney disease. The $24 \mathrm{~h}$ sample (24S) and the second morning sample (SMS) were collected from each patient. Patients were divided in two groups according to urinary concentrations of alpha-1-microglobulin (A1M), which was used as tubular marker. Urinary albumin and A1M were measured by immunonephelometric assay (Behring nephelometer), and creatinine by a kinetic alkaline picrate method (ARCHITECT ci8200).

Results: The median (range) urinary concentrations of albumin were 777.6 (4.44-15380) $\mathrm{mg} /$ day in $24 \mathrm{~S}$ and $67.84(0.46-1470.6) \mathrm{mg} / \mathrm{mmol}$ creatinine in SMS. Albumin values, in both urine samples, were higher in group with elevated A1M than in group with normal A1M. Albumin values in 245 highly correlated $(P<0.001)$ with values in SMS $(r=0.940)$. In addition, the correlation coefficients between albumin levels from different urine samples in the groups with normal and elevated A1M (0.955 vs. 0.920) didn't differ significantly. The mean difference (SD) value, from Bland-Altman analysis, between two urine samples (all albumin concentrations expressed as $\mathrm{mg} / \mathrm{mmol}$ creatinine) was $-12,8 \%(36,9 \%)$.

Conclusion: The results from this study support the use of albumin-to-creatinine ratio on random or morning spot samples as a convenient and suitable alternative to the $24 \mathrm{~h}$ collection.

Key words: albuminuria; second morning sample; 24h sample

*Corresponding author: mdajak@sbb.rs 
P8

\section{Serbian survey study on extra-analytical phase of laboratory diagnostics}

\author{
Šumarac $Z^{* 1}$, Beletić $A^{1}$, Ignjatović $S^{1,2}$, Lalić $N^{1}$, Milinković $N^{1}$, \\ Majkić-Singh $\mathrm{N}^{1,2}$ \\ ${ }^{1}$ Institute for Medical Biochemistry, Clinical Centre of Serbia, \\ Belgrade, Serbia \\ 2Pharmaceutical Faculty, University of Belgrade, Belgrade, \\ Serbia
}

Background: Pre-analytical and post-analytical phases have become the major source of laboratory errors. Aiming to evaluate the situation in the extra-analytical phase in medical laboratories in Serbia, a survey study was conducted in 2012, among the members of Serbian Chamber of Medical Biochemists (SCMB).

Materials and Methods: An anonymous questionnaire was handed out to 510 members of SCMB. It consisted of 24 questions divided in groups according to the following issues: preparation of patients for laboratory investigation, identification of patients, requesting laboratory analysis, techniques in sampling venous and capillary blood, sample handling, managing samples with interferences, reporting tests results and keeping records according to the ISO 15189 standard. Average score for each group and the overall score was calculated using the four grade Likert scale (never-1, rarely-2, often-3 and always-4).

Results: $47 \%$ of participants completed the questionnaire $(62 \%$ from primary health care laboratories, $23 \%$ from specialized hospitals and clinical laboratories and 15\% from laboratories of university hospital, $10 \%$ from private laboratories). The average overall score was $2.69 \pm 0.62$ (mean \pm standard deviation). Questions on managing samples with interferences achieved the lowest score $(2.52 \pm 0.79)$, while the questions about sample handling had the highest score $(3.09 \pm 0.49)$. Significant differences in scores pertaining phlebotomy procedure and requesting laboratory analysis were achieved between private and state laboratories ( $P=0.002, P=0.007$, respectively). $51 \%$ of laboratories always record turn-around-time, but $33 \%$ of laboratories never record non-conformities in laboratory practice.

Conclusions: Analysis of obtained data revealed a high degree of variability in some extra-analytical processes among Serbian laboratories. Results highlight the urgent need for further improvement through adopting and implementation of standardized procedures, as well as better continuing education for all laboratory personnel in the field of extra-analytical phase.

Key words: laboratory diagnostics; extra-analytical phase; survey study

*Corresponding author: zsumarac@gmail.com

\section{P9}

\section{Effects of hemolysis, lipemia and bilirubinemia on acute phase protein levels in bovine serum samples}

\author{
Akay B, Ulutaş PA*
}

Adnan Menderes University, Faculty of Veterinary Medicine, Department of Biochemistry Işıklı, Aydın, Turkey

Backround: Acute phase response is an early and complex reaction of an animal against a variety of injury, infection, trauma or malignancy. The rapid increase in the concentrations of those proteins alter a tissue injury, making them useful marker of inflammation for clinical diagnosis, prognosis and treatment monitoring. The aim of this study was to determine the effects of hemolysis, lipemia and bilirubinemia for haptoglobin, serum amyloid $A$ and ceruloplasmin levels those of which are commonly used assays in cattle practice.

Materials and methods: Serum samples were withdrawn from clinically normal cattle. Serum samples were prepared, divided into 4 portions and then stored on $-20{ }^{\circ} \mathrm{C}$. Serum samples were obtained within healthy cattle. Solutions of haemoglobin, lipid and bilirubin were added to serum samples. Lipid and bilirubin concentrations were determined by commercial test kits (Archem, Tur- 
key) using photometer (Sinnova, BS-3000P, China). Haemoglobin concentration in hemolysate was confirmed by analyses on UV-spectrophotometer (Schimadzu UV-1601, Japan) via cyanmethaemoglobin. Ceruloplasmine concentrations were determined in spectrophotometer. Serum concentrations of haptoglobin and SAA concentrations were determined commercial test kits (Haptoglobin kit, SAA kit, Tridelta Development Ltd., Greystones, Ireland) using microtiter plates and an ELISA reader (Anthos 2010, Anthos Labtec Instruments, Salzburg, Austria).

Results: All acute phase protein analyses had effect on the haemoglobin, lipid and bilirubin added. Haptoglobin concentration was higher in hemolysed and hyperbilirubianemic samples. Haptoglobin levels were significantly elevated in lipemic samples in contrast to normal samples. SAA concentrations were significantly effected in bilirubinemic sera samples. Ceruloplasmin analysis results were changed only in haemolysed samples.

Conclusion: In conclusion it should be suggested that hemolysis, lipemia and bilirubienemia had apparent effect on the acute phase protein analysis at various severity. This relevant data might be useful for future analysis in cattle.

Key words: hemolysis; lipemia; bilirubinemia; acute phase proteins

*Corresponding author: paulutas@adu.edu.tr

\section{P10}

\section{Evaluation of BD preset and BD A-line critical care syringes with other currently marketed devices for ionized calcium, potassium, sodium and $\mathrm{pH}$}

\author{
Church S*1, Plokhoy E2 , Rosa R², Stankovic A \\ 'BD Diagnostics, Preanalytical Systems, Oxford Science Park, \\ Oxford, UK \\ ${ }^{2}$ BD Diagnostics, Preanalytical Systems, Franklin Lakes, NJ, USA
}

Background: Blood gas analysis is essential for patient care and provides detailed information re- garding cardiopulmonary and metabolic homeostasis. BD Preset and BD A-line syringes contain calcium balanced lithium heparin spray dried on the sides of the syringe and can be used to perform critical care tests. Syringes were evaluated at 15 and 60 minutes, typically the times for stat testing.

Materials and methods: Clinical performance of BD Preset and BD A-line critical care syringes was compared with Sarstedt Blood Gas Monovette and Radiometer Pico 50 syringes for the testing of ionized calcium (iCa), potassium $(\mathrm{K})$, sodium $(\mathrm{Na})$ and $\mathrm{pH}$. Venous specimens were collected from 30 adult subjects into each syringe. Testing was performed at 15 and 60 minutes post blood collection and analyzed on the Siemens RAPIDPoint. Mean between-syringe biases with $90 \%$ simultaneous intervals (for $95 \%$ two one-sided test) were calculated for the syringe comparisons per time interval. Clinical criteria for the bias - the maximum allowable difference in test results-were defined for each comparison and analyte. Equivalence was established if the mean bias and 95\% limits were within the clinical criteria.

Results: Clinical equivalence was demonstrated for all comparisons except those listed below. Mean biases were within the clinical criteria, but the $95 \%$ limits exceeded the criteria. Following data review, the results were considered clinically acceptable. Comparison/analyte [clinical criteria, mean bias (95\% confidence limits)]: BD Preset and BD A-line vs. Sarstedt at 60 minutes/ $\mathrm{Na}[3.0$ $\mathrm{mmol} / \mathrm{L}, 1.55(0.03,3.06) ; 1.60(0.08,3.11)]$, respectively; BD Preset and BD A-line vs. Radiometer at 60 minutes $/ \mathrm{pH}[0.03$ units, $-0.0104(-0.0337,0.0129)$; $-0.0130(-0.0363,0.0104)]$, respectively; BD Preset vs. Radiometer at 15 and 60 minutes/iCa [0.05 $\mathrm{mmol} / \mathrm{L},-0.021 \quad(-0.051,0.009) ;-0.023 \quad(-0.053$, 0.007)], respectively.

Conclusion: Clinical equivalence or acceptability was demonstrated for all analytes for each syringe comparison at both time intervals.

Key words: blood gases; critical care syringes; lithium heparin

*Corresponding author: Stephen_CHURCH@europe.bd.com 
P11

\section{Cardiovascular diseases predicted by fasting and nonfasting lipids and apolipoproteins}

\author{
Kolpepaj R*1, Buzo S2, Harxhi A ${ }^{3}$, Kallco M ${ }^{4}$ \\ ${ }^{1}$ University of Tirana, Faculty of Nursing, Tirana, Albania \\ 2Laboratory Department, University Hospital Centre "Mother \\ Teresa", Tirana, Albania \\ 3University Hospital Centre "Mother Teresa", Tirana, Albania \\ ${ }^{4}$ Hospital Regional, Durres, Albania
}

Background: Most humans are in the nonfasting or postprandial state in the majority of a 24 hour cycle; however, lipids, lipoproteins, and apolipoproteins are usually measured in the fasting state. Although guidelines recommend measuring fasting lipids for initial screening of adults without cardiovascular disease (CVD), recent studies suggest that nonfasting triglycerides may be superior to fasting. Recently, a strong association was found between elevated levels of nonfasting triglycerides, which indicate the presence of remnant lipoproteins, and increased risk of ischemic heart disease.

Methods and results: Of the 223 participant healthy women in the prospective study (149 fasting; 74 nonfasting), 72 participant developed CVD (48 fasting; 24 nonfasting). Participants were examined over 2 -years follow-up. Lipid concentrations differed minimally $(<5 \%)$ for fasting versus nonfasting (except for triglycerides). However, stronger associations with CVD were noted for fasting total cholesterol (adjusted fasting hazard ratio $[\mathrm{HR}$ ], 1.12 per $1-\mathrm{SD}$ increment; $96 \% \mathrm{Cl}$, 1.121.28; nonfasting $\mathrm{HR}, 1.07 ; 96 \% \mathrm{Cl}, 0.93-1.12)$, lowdensity lipoprotein (LDL) cholesterol (fasting $H R$, 1.12; $96 \% \mathrm{Cl}$, 1.13-1.29; nonfasting $\mathrm{HR}, 1.00 ; 96 \% \mathrm{Cl}$, 0.87-1.15), apolipoprotein B-100 (fasting HR, 1.36; $96 \% \mathrm{Cl}, 1.27-1.45$; nonfasting $\mathrm{HR}, 1.20 ; 96 \% \mathrm{Cl}, 1.05-$ 1.36), non-high-density lipoprotein (HDL) cholesterol (fasting $\mathrm{HR}, 1.29 ; 96 \% \mathrm{Cl}, 1.21-1.38$; nonfasting $\mathrm{HR}, 1.15 ; 96 \% \mathrm{Cl}, 1.01$ to 1.31 ), and apolipoprotein B-100/A-1 ratio (fasting $\mathrm{HR}, 1.39 ; 96 \% \mathrm{Cl}, 1.30$ to 1.48; nonfasting $\mathrm{HR}, 1.18 ; 96 \% \mathrm{Cl}, 1.09$ to 1.27 ). Compared with fasting levels, nonfasting HDL cho- lesterol, apolipoprotein A-1, and total/HDL cholesterol ratio had similar associations, and triglycerides had a stronger association, with CVD. Significant interactions were seen for LDL cholesterol and apolipoprotein B-100/A-1 ratio with fasting status ( $P$ for interaction $=0.03$ and $<0.001$, respectively).

Conclusions: In this study population is demonstrated that HDL cholesterol, triglycerides, total/ HDL cholesterol ratio, and apolipoprotein A-1 predict CVD when measured nonfasting. On the other side, total, LDL, and non-HDL cholesterol, in addition to apolipoprotein B-100 and B-100/A-1 ratio, provide less useful CVD risk information when nonfasting, despite small changes in their concentrations. Guidelines for lipid screening may need to consider these differences.

Key words: triglyceride; cholesterol; fasting; apo B-100

*Corresponding author: rkolpepaj@yahoo.com

\section{P12}

\section{Evaluation of preanalytical errors in prothrombin time tests including hemolysis, lipemia, icterus and insufficient or clotted specimens}

\author{
Djordjevic A*1, Milinkovic N1, Dopsaj V1,2, Ignjatovic S1,2 \\ ${ }^{1}$ Center of Medical Biochemistry, Clinical Center of Serbia, \\ Belgrade, Serbia \\ 2Institute of Medical Biochemistry, Faculty of Pharmacy, \\ University of Belgrade, Belgrade, Serbia
}

Background: The presence of interfering substances, clots, and inappropriate blood/anticoagulant ratio in samples are a critical preanalytical problem. The aim of this study was to evaluate the number of prothrombin time tests (PT) that were performed or rejected for analysis due to influence of preanalytical variables.

Material and methods: All PT tests were performed with Thromborel $^{\circledR}$ S reagent on BCS $^{\circledR}$ XP (Siemens) on two wavelengths: $405 \mathrm{~nm}$ and 570 
$\mathrm{nm}$ (for icteric, hemolytic or lipemic samples) and were reported in seconds and INR. Free hemoglobin in plasma was measured on Beckman Coulter LH750 hematology analyzer. Concentrations of triglycerides and bilirubin were measured on Architect ci8200 (Abbott).

Results: Total number of blood samples for PT was 6969. We identified 59 hemolytic specimens $(0.84 \%)$ with concentration of hemoglobin in plasma: < $1 \mathrm{~g} / \mathrm{L}(45 \%)$, between 1-2 g/L (29\%), > $2 \mathrm{~g} / \mathrm{L}$ $(26 \%)$, and $>6 \mathrm{~g} / \mathrm{L}(1 \%)$, respectively. Total number of lipemic samples was $14(0.20 \%)$ : with triglycerides $<3.38 \mathrm{mmol} / \mathrm{L}(71 \%),<4.58 \mathrm{mmol} / \mathrm{L}(14 \%)$ and $>4.58 \mathrm{mmol} / \mathrm{L}(15 \%)$, respectively. We found 13 icteric samples (0.19\%): with bilirubin $<205.2 \mu \mathrm{mol} / \mathrm{L}$ (62\%) and $>205.2 \mu \mathrm{mol} / \mathrm{L}$ (38\%). We visually identified 24 clotted samples (0.34\%) and 16 samples with inappropriate volume $(0.23 \%)$. Correlation between PT determined on $570 \mathrm{~nm}$ and PT from repeated samples of the same patients on $405 \mathrm{~nm}$ without interferences, was $r=0.91(P<0.05)$. Total number of rejected samples was 43 (0.62\%): $2.3 \%$ due to hemolysis, $4.7 \%$ due to lipemia, $37.2 \%$ due to inappropriate volume and $55.8 \%$ due to clotting.

Conclusion: Overall PT tests with interferences $126(1.8 \%)$ we analyzed 83 tests (1.2\%) on $570 \mathrm{~nm}$. Significant percentage of rejected samples $93 \%$ was due to inappropriate venepuncture and inadequate filling of the test tube.

Key words: hemolysis; preanalytic variables; prothrombin time

*Corresponding author: anica57djordjevic@gmail.com
P13

\section{Effects of pneumatic tube delivery system on blood routine biochemical parameters and intact parathyroid hormone}

Deveci K*, Sancakdar E, Aydın H, Erkoç F, Uysal EB, Gokakin AK

Department of Biochemistry and Clinical Biochemistry, Ataturk Training Hospital, Izmir, Turkey

Introduction: Pneumatic tube systems (PTSs) are widely used in hospitals for convenience and reduced turnaround times. The aim of this study was to investigate the effects of PTS on routine biochemistry and intact parathyroid hormone (iPTH) analysis.

Materials and methods: In this study, we used blood samples from 20 healthy volunteers. Three vacutainer tubes of blood were taken from each volunteer. These samples were sent to the central laboratory from two different locations by PTS, and a human porter. Maximum distance location (Group I) and the minimum distance location (Group II) via the PTS and a porter (Group III). Routine biochemical parameters and PTH levels in serum samples of three groups were measured.

Results: The bicarbonate and lactate dehydrogenase (LD) levels were significantly higher in samples transported via PTS than samples transported via a porter. The bicarbonate and LD levels were significantly higher in the Group I than Group II. No statistically significant differences between PTS samples and samples delivered by hand were observed in other routine biochemical parameters, and in iPTH levels.

Conclusion: The transport of non-centrifuged samples via PTS, dependent on PTS length, may affect serum bicarbonate and LD levels.

Key words: pneumatic tube systems; bicarbonate; lactate dehydrogenase; intact parathyroid hormone

*Corresponding author: drkdeveci@gmail.com 


\section{P14}

\section{The incidence of macroprolactinemia in patients with hyperprolactinemia}

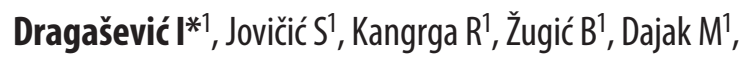 \\ Ignjatovićs ${ }^{2}$
}

\section{P15}

\section{Six Sigma in the preanalytical phase for continuous quality Improvement in clinical laboratories}

\author{
Iren Emekli D, Aslan D*, Zorbozan N \\ Pamukkale University, Faculty of Medicine, Department of \\ Medical Biochemistry, Denizli, Turkey
}

Background and aim: The preanalytical (Pre-A) phase is responsible $46-77 \%$ of total errors in the total testing process. Every laboratory should have a policy for detection and prevention of errors. The frequency of errors should be determined systematically in the standardized manner. Six Sigma (6S) is a process improvement set of tools and strategies which the defects (errors) can be identified and eliminated in a process. By estimating the sigma metric for each error in the Pre-A phase, the most common errors can be determined. In this study, we aimed to assess the preanalytical process performance according to six sigma methodology.

Materials and method: We determined the most common possible Pre-A errors (sample-improperly labeled; sample-hemolyzed; sample-improper transport; sample-insufficient; sample-damaged; sample-clotted; information (clinical)-insufficient; order-incorrect; order-duplicate; container-inappropriate). We standardized the terminology and structured the reporting system in our Hospital Information System (HIS) and Laboratory Information System (LIS). Data were collected monthly for the period of January 2012 - June 2012; analyzed, and sigma metrics were calculated for each error by using formula "NORMSINV [1 - (total defects/total opportunities)] $+1.5^{\prime \prime}$ in the Microsoft Excel Spreadsheet. We have chosen the quality performance as 4.6 sigma which shows $10 \%$ waste with 1000 DPM.

Results: Sigma metrics calculated for the samplehemolyzed, sample-clotted, sample-insufficient, and sample-improperly labeled were under 4.6.

Conclusion: Each type of error can be sequenced according to its sigma metric, hence the error which is the most common and causes the waste- 
ful operations with the risk to the patients can be identified and the error sources can be improved. As used in the industry and shown in the several studies the six sigma approach is invaluable tool for continuous quality improvement in clinical laboratories but the HIS and LIS should be structured for error collection and also the competency of laboratory staff should be improved for usage of six sigma tools.

Key words: quality in laboratory medicine; clinical laboratory; preanalytical errors; patient safety; six sigma

*Corresponding author: daslan@pau.edu.tr

\section{P16}

\section{Quality Indicators in preanalytical phase for continuous quality improvement in clinical laboratories}

Iren Emekli D, Aslan D*, Zorbozan N

Pamukkale University, Faculty of Medicine, Department of Medical Biochemistry, Denizli, Turkey

Background and aim: The preanalytical (Pre-A) phase is responsible $46-77 \%$ of total errors in the total testing process. Every laboratory should have a policy for detection and prevention of errors. The frequency of errors should be determined systematically in the standardized manner. In this study we aimed to establish a system in our laboratory for collecting and evaluating the Pre-A errors according to the Quality Indicators (QIs) developed by the IFCC Working Group on "Laboratory Errors and Patient Safety" (WG-LEPS).

Materials and methods: We determined the most possible Pre-A errors (sample-improperly labeled; sample-hemolyzed; sample-improper transport; sample-insufficient; sample-damaged; sampleclotted; information (clinical)-insufficient; order-incorrect; order-duplicate; container-inappropriate). We standardized the terminology and structured the reporting system in our Hospital Information System (HIS) and Laboratory Information System (LIS). Data were collected monthly for the period of January 2012-June 2012; analyzed, and the Qls were calculated. The results were evaluated according to the desirable performance levels proposed by the IFCC WG-LEPS.

Results: The Qls for "sample-hemolyzed" and "sample-clotted" had showed that these errors should be considered primarily in the quality improvement studies.

Conclusion: Our results showed that quality indicators may be useful for evaluation of Pre-A process especially for the determination of errors highly occurred. But the HIS and LIS should be structured for error collection and also the competency of laboratory staff should be improved for data management, and usage of the quality control tools.

Key words: quality in laboratory medicine; clinical laboratory; preanalytical errors; patient safety; quality indicator

*Corresponding author: daslan@pau.edu.tr

\section{P17}

\section{An underestimated preanalytical error source: centrifuge temperature}

\author{
Y.lmaz FM1,2, Kıral S*1, Boğdaycıoğlu N1, Uysal S1 \\ ${ }^{1}$ Ankara Numune Education and Research Hospital Clinical \\ Biochemistry Laboratory, Ankara, Turkey \\ ${ }^{2}$ Yıldırım Beyazıt University Medical Faculty, Clinical \\ Biochemistry Department, Ankara, Turkey
}

Background: The study demonstrates a gel problem sourced from the internal heat of centrifuges without temperature control. It was planned to investigate the internal temperatures of temperature controlled centrifuges and the centrifuges without temperature control and the effect of centrifuge temperature on enzymes and thyroid panel.

Materials and methods: Internal heat of the centrifuges was monitored in the busiest working hours. Blood samples were obtained from 42 patients to two separate biochemistry tubes. One sample of the same patient was centrifuged with a temperature-controlled centrifuge (Group 1) and 
the other was centrifuged with a centrifuge without temperature control (Group 2). AST, ALT, GGT, LD, total protein, albumin, TSH, fT3 and fT4 levels were determined in the same run of the analyzer.

Results: ALT and TSH values in Group 2 were significantly lower; fT4 values were significantly higher than Group 1.

Conclusions: Laboratories should be aware of a possible internal heat production in centrifuges without temperature control and gel tubes should be centrifuged in temperature controlled centrifuges especially in centrifuge units with high working load.

Key words: centrifuge; temperature-controlled centrifuge; gel tube; interference

*Corresponding author: serkankiral71@hotmail.com

\section{P18}

\section{Examination of the impact of lipemia in determining the level of hemoglobin}

\author{
Vukosavljević D*, Dajak M, Stjepanović V, Đorđević A, Šumarac Z \\ Center of Medical Biochemistry, Clinical Center of Serbia, \\ Belgrade, Serbia
}

Background: It is well known that lipemia interferes in analyzing some of the hematologic parameters. Turbidity of the sample has impact on spectrophotometric determining of the hemoglobin. Different levels of triglycerides (cut off values) which interfere in determining the hemoglobin, erythrocyte indexes (MCH, MCHC) and other hematologic parameters have been published.

Materials and methods: The study included 125 patients with high levels of triglycerides (TG) who were divided in 3 groups- A (TG: $3-7 \mathrm{mmol} / \mathrm{L}$ ), B (TG: 7-12 mmol/L), C (TG: > $12 \mathrm{mmol} / \mathrm{L}$ ). Hemoglobin was determined before and after the treatment of EDTA-sample. The treatment of the EDTA-sample implied "saline replacement" which included whole blood centrifugation, replacement of the plasma with the same volume of saline, mixing the sample and its analyzing. Hemoglobin was deter- mined using spectrophotometric method on the HMX-Al analyzer (Beckman Coulter, Germany).

Results: Considering the whole examined population, significant difference in correlation of the level of hemoglobin before and after the treatment of the sample has not been found. Mean values of hemoglobin in groups $A, B$ and $C$, before the treatment, are 117.8, 121.2 and $150 \mathrm{~g} / \mathrm{L}$ and after the treatment, are 116.9, 118.6 and $142.2 \mathrm{~g} / \mathrm{L}$, respectively. In the groups A and B significant difference in correlation of the level of hemoglobin before and after the treatment has not been found $(P>$ $0.05)$, while in group $C$ has been found $(P=0.002)$.

Conclusion: Results of this study have shown that the levels of triglycerides $>12 \mathrm{mmol} / \mathrm{L}$ do impact on determining the level of hemoglobin using spectrophotometric method because of the significant turbidity of the sample.

Key words: haemoglobin; triglycerides; spectrophotometric method

*Corresponding author: dvukosavljevic@hotmail.rs

\section{P19}

\section{NIDCAP - how biomedical scientists can contribute with expertise in phlebotomy and preanalysis on neonates}

\section{Viddal BV}

NITO The Norwegian Institute of Biomedical Science, Biomedical Scientist, Ålesund, Norway

Background: NIDCAP, Newborn Individual Development Care and assessment Program, is an instrument for ensuring giving best possible care for the neonate born prior to week 34 , but it also focuses on how the baby can be protected against light, sounds and pain.

Materials and methods: This is an area where the Biomedical Scientist (BMS), can help supervise nurses and doctors and teach them possible sources of preanalytical errors. The NIDCAP method states that treatment should be adapted to suit each individual baby. Blood samples should there- 
fore be taken when it suits the baby and not necessarily when it suits the phlebotomist. To ease pain, the baby is given glucose orally approximately two minutes before the procedure and samples are taken by venipuncture. Venipuncture gives a better flow of blood which leads to fewer preanalytical errors such as haemolysis, clots of platelets and contamination of extracellular fluid.

Results: Better quality of the sample material leads to an improved reliance of the test results. This again leads to fewer samples taken, something that is ideal with this programme. By always evaluating every individual case, hospitals can reduce the amount of samples by up to 70-80 per cent. This was the case in Ålesund after NIDCAP was introduced in 1997. From 1997 to 2005 the amount of samples was reduced with 70 percent. From 2005 to 2010 there has been a small increase, but still the amount of samples per patient is held low.

Conclusion: The BMS should help support NIDCAP by adapting sampling routines and methods when dealing with premature babies. By co operating with the staff at the premature baby wards we can contribute with our expertise and improve treatment of the little neonate.

Key words: NIDCAP; preanalysis; neonatal; phlebotomy

Corresponding author: brit.valaas.viddal@nito.no

\section{P20}

\section{How is phlebotomy organised in Norwegian hospitals?}

\section{Viddal BV, Roald MN*}

NITO The Norwegian Institute of Biomedical Science, Oslo, Norway

Background: Many studies document that the majority of mistakes in an analytical process are made during the pre-analytical phase. Recent studies show that laboratory personnel make fewer mistakes when taking blood samples than other health personnel. In Norwegian hospitals phlebotomy has traditionally been centralised to the medical laboratories and to a large extent carried out by biomedical laboratory scientists from medical biochemistry departments.

Material and methods: A few hospitals have published that they have decentralised part of blood collection to the clinical departments. A questionnaire about the organisation of phlebotomy services was sent to the medical biochemistry department at all Norwegian hospital locations ( $N=53$ ) in August 2011. We asked how phlebotomy is organised in Norwegian hospitals, the reason for the present organisation and asked if more hospitals are planning to decentralise phlebotomy services.

Results: The results were summarized in October 2011. In total 92.5 per cent of the hospitals $(N=49)$ responded to the survey. The results show that of the respondents 44 have centralised organisation of phlebotomy, two hospitals have decentralised phlebotomy and three hospitals have a combined model. The reasons given for centralised blood sampling at the 44 hospital locations have been analysed by a qualitative method, and are shown in the poster.

Conclusion: Most Norwegian hospitals have centralised organisation of phlebotomy, and few are planning to change the way they are organised. The respondents state that the most important reasons for centralised organisation of phlebotomy services are competence and quality aspects, time efficiency and tradition.

Key words: organisation; centralised phlebotomy; decentralised phlebotomy

*Corresponding author: marie.nora.roald@nito.no 


\section{P21}

\section{Influence of metamizole on serum creatinine measurements (case report)}

\author{
Fliser E*, Modrič E, Klavž J, Jevšnikar B, Gorenjak M
}

Department for Laboratory Diagnostics, University Clinical Center Maribor, Maribor, Slovenia

Background: Metamizole is the active substance of an analgesic drug, known as Analgin, Novalgin or Dipyrone. The drug is widely used at medical institutions. We present a case report of metamizole interference on IDMS-traceable enzymatic creatinine assays, which are now recommended for routine measurements by international guidelines.

Materials and methods: A 38-year old man was accepted in emergency department due to injury. Blood samples were taken and serum creatinine below $12 \mu \mathrm{mol} / \mathrm{L}$ was measured with enzymatic method on Siemens Vista analyser. The measurement was repeated on the same instrument and on another Vista instrument with a different reagent lot. All repeated results were below $12 \mu \mathrm{mol} / \mathrm{L}$. The patient received one ampulla of Analgin ( 2.5 gram of metamizole) before blood was drawn, therefore another blood sample was taken afterwards and creatinine of $99 \mu \mathrm{mol} / \mathrm{L}$ was measured.

We monitored 8 different patients after one Analgin ampoule injection to verify metamizole influence. Creatinine was measured before the injection of the drug and after 1, 2, 3, 4 and 6 hours. Measurements were performed with Siemens Healthcare Jaffe- and enzymatic method on Vista analyser and with Roche Diagnostics Jaffe- and enzymatic method applied on Beckman Coulter AU640 Chemistry System.

Results: Creatinine values were below $12 \mu \mathrm{mol} / \mathrm{L}$ with both enzymatic assays 1 hour after Analgin injection in all 8 patients. The values distinguished significantly from the measurement before drug application $(P<0.001)$. After two hours, the enzymatic results were not yet completely comparable to the first measurement $(P=0.016$ Siemens, $P=$ 0.008 Roche), while after three hours and later all measurements were comparable again ( $P>0.050)$.
All creatinine measurements with Siemens Healthcare and Roche Diagnostics Jaffe methods were comparable before and after Analgin injection ( $P$ $>0.050$ ).

Conclusions: Analgin (metamizole) interferes with Roche Diagnostics and Siemens Healthcare enzymatic creatinine assays. We propose measurements with these two assays at least 3 hours after metamizole ampoule injection.

Key words: analgesic drug; interference; enzymatic creatinine assay

*Corresponding author: eva.fliser@ukc-mb.si

\section{P22}

\section{4hr ESR storage stability study}

\author{
Barks T, Robson C, Weir A, Lumley AJ*
}

Sheffield Teaching Hospitals NHS, Sheffield, United Kingdom

Background: The StaRRsed InteRRliner is a fully automated ESR analyser which uses EDTA anticoagulated blood, thereby making use of the full blood count sample. A sample stability study for ESR was undertaken, to assess whether samples stored overnight at GP's surgeries, where best kept at $4^{\circ} \mathrm{C}$ or room temperature, prior to being sent to the laboratory the next day for testing.

Materials and methods: 99 in-patient FBC samples ( $4.0 \mathrm{~mL} \mathrm{~K} \mathrm{~K}_{2}$ EDTA) were selected on the basis of being as fresh as possible and of sufficient blood volume for an ESR test to be performed twice. The study was performed across two sites, Northern General and Royal Hallamshire Hospitals, using identical StaRRsed InteRRliners. All samples had an initial ESR test performed and then the samples were split into 2 groups - 51 were kept at room temperature and 48 were stored at $4{ }^{\circ} \mathrm{C}$. After a period of 24 hours all samples were retested for ESR ensuring that the ones stored in the fridge, had equilibrated to room temperature, prior to testing.

Results: The room temperature samples show a coefficient of determination $R^{2}=0.5078$, whereas 
the $4{ }^{\circ} \mathrm{C}$ show $\mathrm{R}^{2}=0.9719$. Using a two tailed student $t$-test for paired samples the $P$ value was calculated at 0.056 for $4{ }^{\circ} \mathrm{C}$ samples and $<0.001$ for RT samples.

Conclusion: By comparing the room temperature and storage at $4{ }^{\circ} \mathrm{C}$ samples, it showed ESR results are grossly affected by storage at RT, but show minimal change after storage at $4^{\circ} \mathrm{C}$ for 24 hours. It was concluded that samples are best stored overnight in the fridge at the GP surgery if they are taken too late in the day to be collected by the vans and brought to the laboratory for testing.

Key words: 24hr; ESR; storage; stability

*Corresponding author: andrew.lumley@sth.nhs.uk

\section{P23}

\section{Test request variability in primary care in Catalonia}

Ibarz $\mathbf{M}^{* 1}$, Perich C ${ }^{2}$, Llopis $\mathrm{MA}^{3}$, Alvarez $\mathrm{V}^{4}$, Domenech MV ${ }^{5}$, Pastor $\mathrm{R}^{6}$, Busquets $\mathrm{G}^{7}$, LLovet Ml ${ }^{8}$, Simon $\mathrm{M}^{9}$, Biosca $\mathrm{C}^{10}$

\footnotetext{
${ }^{1}$ Laboratori Clínic Hospital Arnau de Vilanova, Lleida, Spain 2Laboratori Clínic Bon Pastor, Barcelona, Spain

${ }^{3}$ Laboratori Clínic Barcelonès Nord i Vallès Oriental, Badalona, Spain

4Laboratori Clínic L'Hospitalet, L'Hospitalet de Llobregat, Barcelona, Spain

${ }^{5}$ Laboratori Clínic Manso, Barcelona, Spain

${ }^{6}$ Laboratori Clínic Hospital Joan XXIII, Tarragona, Spain

${ }^{7}$ Laboratori Clínic Hospital Dr. Josep Trueta, Girona, Spain

${ }^{8}$ Laboratori Clínic Hospital Verge de la Cinta, Tortosa, Spain

${ }^{9}$ Consorci de Laboratori Intercomarcal Anoia, Penedés i Garraf,

Vilafranca del Penedés, Spain

${ }^{10}$ Servei Bioquímica Clínica Hospital Germans Trias i Pujol, Badalona, Spain
}

Background: Test request variability is an important performance indicator for preanalytical quality and laboratory efficiency. To know this variability is the first step to improve the use of clinical tests.

The aim of this study was to describe and evaluate the differences and possible determining factors of test request variability in primary care in a group of laboratories belonging to the public network of the Catalonian Public Health Service.

Materials and methods: The group is formed by ten laboratories, four provide services exclusively to primary care and six to hospitalized and primary care patients. Statistics of 2011 were obtained and the number of tests was set to the number of primary requests for every laboratory. The median and percentiles ratios were calculated. Possible determining factors were studied.

Results: 2.815 .563 primary care requests were processed with a total of 32.706 .843 analytical tests. The 15 most frequently ordered tests accounted for $60 \%$ of the laboratory activity and they were in descending order of frequency (depending on the median of the ratios of the different laboratories): cell blood count, glucose, cholesterol, creatinine, alanine aminotransferase, triglycerides, HDL-cholesterol, gamma-glutamiyltranspeptidase, uric acid, electrolyte profile (sodium and potassium), urinalysis, bilirrubine, thyrotropin, aspartate aminotransferase and alkaline phosphatase. Median ratios for all laboratories were within the interquartile range for the 7 first ones of the list and for thyrotropin as well.

Conclusions: These results show that the test ordering is fairly homogeneous in primary care in Catalonia, surely as consequence of the application of agreed protocols from 1997 for several pathologies and health states. The discrepant results could be due to diverse adaptations of protocols and/or diagnostic algorithms in laboratories and different specialists' integration in primary care.

Key words: quality; preanalytical-phase; test-requiring

\footnotetext{
*Corresponding author: mibarz.lleida.ics@gencat.cat
} 
P24

\section{Assessment of the preanalytical phase for molecular biology testing through participation in EU SPIDIA project}

\author{
Kardum Paro MM*, Šiftar Z, Flegar- MeštrićZ
}

Department of Medical Biochemistry and Laboratory Medicine, „Merkur" University Hospital, Zagreb, Croatia

Background: In molecular biology testing definite gene changes are identified or ruled out directly or indirectly on nucleic acids level. Because of variation of molecular biology testing across Europe, inter-laboratory comparability is possible through methodological proficiency testing (PT) programs intended for monitoring and controlling the quality of the preanalytical phase or External Quality Assurance (EQA) programs intended for relatively frequently tested diseases. To monitor and evaluate the laboratory preanalytical phase of molecular biology testing, we joined to the SPIDIA-DNA trial of the European Union (EU) project Standardisation and improvement of generic pre-analytical tools and procedures for in-vitro diagnostics (SPIDIA).

Materials and methods: In the $2^{\text {nd }}$ SPIDIA- DNA trial the same whole blood sample was send to 174 participants to perform the DNA extraction using their own protocol and reagents. The participants were asked to make the DNA UV analysis and calculations, report results and additional information on the web site and to send back extracted DNA for further SPIDIA DNA analysis. The laboratory preanalytical phase of DNA extraction SPIDIA evaluated through DNA Quality and DNA Quantity by spectrophotometric method, DNA Integrity by ad-hoc algorithm combining by-eye evaluation of a panel of experts and Image J software through three categories (high, intermediate and low) and PCR kinetics interferences by real-time PCR using RnaseP and Kineret software. Final SPIDIA- DNA report included statistical analysis and the results interpretation in a form of a score which included the comparison of single laboratory performance with that of other participants.
Results: Statistical results of the DNA Quality and DNA Quantity $(\mathrm{N}=174)$ by spectrophotometric method were 1,810 (lab) vs. 1,857 (SPIDIA) and 27,820 (ng/ $\mu \mathrm{L}$; lab) vs. 27,200 (ng/ $\mu \mathrm{L} ;$ SPIDIA). Results were scored and interpreted as "in control". Only DNA Integrity $(\mathrm{N}=158)$ was scored as of "intermediate integrity". PCR kinetics evaluation ( $\mathrm{N}=$ 173) showed value under the 5,99 SPIDIA threshold and score interpreted as "in control“.

Conclusions: Overall SPIDIA- DNA statistics objectively provides deep monitoring of the preanalytical phase of molecular biology testing. Results interpretation reflects state of the art in laboratory performance so that special emphasis could be placed on laboratories with sub- optimal performance.

Key words: SPIDIA; preanalytical phase; molecular biology testing

*Corresponding author: mirjanamariana@gmail.com

\section{P25}

\section{Frequency of lipemia and its preanalytical impact on iron determination in pediatric population - our experience}

\author{
Lenicek Krleza J*, Rajcic A, Grzunov A \\ Children's Hospital Zagreb, Department of Laboratory \\ Diagnostics, Zagreb, Croatia
}

Introduction: Samples for iron measurement should be taken in the morning from patients in a fasting state and there can be significant interference from lipemia. As most of our hospital clinics operate until the late afternoon hours, requests for iron determination pour in throughout all day.

Material and methods: The aim of this study was to determine the amount of lipemic samples accepted for iron determination in our laboratory over a period of three months. All samples were visually inspected for turbidity. For 41 lipemic samples time of sampling and patient age were noted. Iron concentration was determined two times in 
all lipemic sera. The first measurements from the original samples, and the second after the same samples were diluted 1:2 with normal saline. The iron level was determined on Olympus AU400 with a method for serum iron using TPTZ\{2,4,6-Tri-(2pyridyl)-5-triazine as the chromogen. To compare the values of iron measured before and after dilution with saline nonparametric Wilcoxon test for paired data was used.

Results: $13 \%$ of samples were found visibly turbid. For $44 \%$ of lipemic samples sampling time was after 10 a.m. $71 \%$ of patients whose samples were lipemic were under 2 years of age. For $37 \%$ of lipemic sera a decision was made that results obtained from diluted samples are to be issued. Wilcoxon test did not show a significant difference between the two measures ( $p=0.414$ ).

Conclusion: Turbidity was observed in a significant number of samples. In a sizable number of cases, the results obtained from diluted samples were issued. Almost half of the lipemic samples were taken after 10 a.m., and it is very likely that the cause of lipemia is food consummation. The actual iron level in these patients is questionable, since iron values decrease by $30 \%$ during the course of the day.

Key words: child; iron; lipemia; sampling time; interference

*Corresponding author: jlenicek@gmail.com

\section{P26}

\section{Preanalytical variability of the measurement of alpha fetoprotein in amniotic fluid: case report}

\author{
Brescia V*, Scolozzi S, Circhetta S
}

U. O. C. Medicina di Laboratorio, A.O. Cardinale G. Panico

Tricase, Lecce, Italy

Background: To evaluate a case of increased alpha-fetoprotein in amniotic fluid (AF-AFP) without ultrasonographic evidence of open neural tube defects (NTD) or other fetal abnormalities.
Materials and methods: A 38-year-old (age-specific risk) pregnant woman referred mild blood loss at the end of the third month of pregnancy; genetic amniocentesis and AFP-AP assay were performed at the 16th week. AF showed slight blood contamination. A primary aliquot of $2 \mathrm{~mL}$ AF was centrifuged at $1200 \mathrm{rpm}$ within $3 \mathrm{hrs}$ of withdrawal. AFP-AF assay was done with an automatic DxC880i Beckman Coulter (Chemiluminescent Immunoassay). The corpuscular part of the AF was washed with physiological solution, recentrifuged and lysed with redistilled water for HBF assay in high-performance liquid chromatography HPLC (Variant II BIORAD). The second ultrasound screening was negative for NTD or other fetal abnormalities (18th week).

Results: AFP-AP was $32,4 \mu \mathrm{g} / \mathrm{mL}(>2.0<2.5 \mathrm{MoM}$ ), HBF on lysed AF blood was positive for fetal bleeding (16\%). The AFP-AF findings were integrated with the warning "contamination by fetal blood: AFP-AF value not specific for NTD". The pregnancy outcome was physiological.

Conclusion: The AFP-AF test is useful for screening for NTD or other fetal abnormalities. There is a high AFP concentration gradient between fetal plasma and AF. The presence of fetal bleeding in the AF can cause false positive increases in AFP-AF. The fetal hemoglobin assay in HPLC we adopted was shown to be reliable and not influenced by a different matrix. Therefore, in our laboratory all AF specimens testing positive for AFP (> 2.0 MoM) will also be tested for the presence of HBF to minimize errors in NTD risk estimation.

Key words: alpha-fetoprotein; amniotic fluid; open neural tube defects

*Corresponding author: v.brescia@piafondazionepanico.it 
P27

\section{Comparison between 6-hour and 24-hour cupriuria in monitoring of therapy with D-penicillamine in patients with Wilson disease}

Ivanova ${ }^{* 1}$, Krastev $Z^{1}$, Petkova $T^{1}$, Dragneva $S^{2}$

1Department of Clinical Laboratory, University Hospital St. I. Rilski, Medical University, Sofia, Bulgaria

${ }^{2}$ Clinic of Gastroenterology, University Hospital St. I. Rilski, Medical University, Sofia, Bulgaria

Background: Adequacy of treatment in patients with Wilson Disease (WD) was monitored by measuring 24-hour urinary copper excretion while on treatment with D-penicillamine. The major effect of the drug is to promote the urinary excretion of copper. Values between 3-8 $\mu \mathrm{mol} / 24 \mathrm{~h}$ (200-500 $\mu \mathrm{g})$ per day cupriuria means adequate therapy. The problems of measuring 24-hour copper excretion include: incomplete urine collection and copper contamination of the collection device. Therefore, by reducing time for testing we will also reduce errors in preanalytical stage of examination.

Materials and methods: The aim of the study was to assess and compare the 6-hour and 24-hour copper urinary excretion in patients with WD treated with D-penicillamine. The data used ware from 42 measurements in 32 patients with proven WD in University Hospital St. Ivan Rilski, Medical University-Sofia. The average dose D-penicillamine was $800-1000 \mathrm{mg}$ per day. Urine was collected in two portions - 6-hour and 18-hour. The concentration of copper in two portions ware calculated to get 24-hour cupriuria. Copper analysis was made with atomic absorption spectrophotometry in Analyst 400 device.

Results: The copper urine excretion in 6-hour, 18hour and in 24-hour urine was respectively $x=4.12$ $\pm 2.26 \mu \mathrm{mol} / 6 \mathrm{~h}, \mathrm{x}=5.75 \pm 3.42 \mu \mathrm{mol} / 18 \mathrm{~h}$ and $\mathrm{x}=$ $9.89 \pm 4.7 \mu \mathrm{mol} / 24 \mathrm{~h}$. Copper excretion in 6-hour urine was $41.7 \%$ of 24 -hour cupriuria.

Conclusion: About half of copper urine excretion was up to 6-hour diuresis. Reducing the time of urine collection could reduce the errors in preana- lytical stage of examination. It is necessary the 6-hour urine copper excretion to be standardized and validated to use it as criteria of adequacy of therapy.

Key words: cupriuria; Wilson Disease; D-penicillamine

*Corresponding author: irena.dimitrova@gmail.com

\section{P28}

The simultaneous effect of closed system urine sampling and the urine stabilizing additive on the outcome of urine sample evaluation

\author{
Lakatos A*, Nagy Cs Zs, Rékási Zs, Magyarlaki T, Kovács LG \\ Clinical Center of the University of Pécs, Laboratory Medicine \\ Institute, Pécs
}

Background: The preanalytical factors have major importance in the evaluation of routine urinalysis and urine sediment examinations as well. The time factor of the preanalytical processes significantly influences the number of urine components of the urine sediment.

Materials and methods: We compared the number of red blood cells, white blood cells and bacteria in samples stored on room temperature, taken by $B D$ Vacutainer ${ }^{\circledR}$ tubes containing urine stabilizing agents (AD), with closed sampling; and on the other hand in samples taken in non-sterile, additive-free plastic tubes (Native).

Results: According to our examinations RBC number significantly decreased after 24 and 48 hours both in the Native tubes and in the BD Vacutainer $^{\circledR}$ evacuated additive containing additive tubes as well. The decrease of WBC number was nearly significant in the Native tubes $(P=0.06 ; 0.18)$, while there were essentially no change in the $B D$ Vacutainer ${ }^{\circledast}$ tubes $(P=0.69 ; 0.80)$. The BACT number increased significantly only in the Native samples.

Conclusions: Our results indicate that preanalytical errors can be lessened with the use of BD Vacu- 
tainer ${ }^{\circledast}$ tubes: the use of this device prevents the change of the number of WBC and BACT as well.

Key words: urine sediment; stabilization; preanalytical

*Corresponding author: agnes.lakatos@aok.pte.hu

\section{P29}

\section{Validation of a pneumatic tube system. Quantification of acceleration forces related to haemolysis.}

\author{
Gómez-Rioja R*, Fernández-Calle P, Alcaide MJ, Iturzaeta JM, \\ Oliver P, Buño A
}

Laboratory Medicine Department, La Paz University Hospital, Madrid, Spain

Background: Pneumatic tube system (PTS) has been described as a cause of in vitro haemolysis. Streichert described a method to measure the total sum of sudden accelerations changes produced during the PTS transport using data-loggers sent along with samples. To validate our PTS, we investigate the quantitative relationship between sudden accelerations changes and their haemolytic effect in healthy volunteers' samples.

Materials and methods: Five plasma samples (lithium-heparin, BD) in the same blood-drawn were obtained from 20 healthy volunteers. One sample was hand transported and considered as the baseline. The rest were sent through the PTS to the laboratory along with the data-logger (MSR 145) repeatedly. One sample was removed prior to each subsequent sending, there being four sendings in total. Once finished, all samples were centrifuged and duplicate measurements of Haemolytic index $(\mathrm{H})$, potassium, AST and LD were performed in a Dimension Vista (Siemens HD). As an index of stress caused by PTS, the accumulative sum of sudden acceleration changes recorded for each sample were used.

Results: A statistically significant relationship was found between the accumulative sum of sudden acceleration changes and haemolysis degree, evi- denced by haemolytic index $(\mathrm{H})$ as well as by plasma increase of intraeritrocitic compounds, being respective Pearson coefficients $r=0.65(H), r=0.53$ (potassium), $r=0.73$ (LD) and $r=0.56$ (AST). The critical threshold to establish a "safe transport "was defined using the slope of the regression equation. The criteria to define a significant change in the test concentration were established following the recommendations of Spanish Society of Clinical Chemistry. Safe threshold were set at $0.69 \times 10^{3}$ $\mathrm{G}(\mathrm{LD}), 1.44 \times 10^{3} \mathrm{G}(\mathrm{AST})$ and $5.08 \times 10^{3} \mathrm{G}$ (potassium).

Conclusion: These results confirm the reliability of Streichert method to validate a PTS. The relation between haemolysis degree and sudden acceleration changes allows the definition of a safe threshold for each test.

*Corresponding author: rgomezr.hulp@salud.madrid.org

\section{P30}

The cost of poor sample quality: assessing the financial impact of sample rejection and recollection in healthcare institutions

Chait $\mathrm{G}^{1}$, Schlueter K2 ${ }^{2}$ Baginska E ${ }^{3}$, Scraba K3 , Flynn L ${ }^{4}$, Church S*5

${ }^{1}$ Whythawk, Oxford, UK

2 BD Diagnostics, Preanalytical Systems, Heidelberg, Germany

${ }^{3}$ BD, Mississauga, Canada

${ }^{4}$ BD Diagnostics, Preanalytical Systems, Franklin Lakes, USA

${ }^{5}$ BD Diagnostics, Preanalytical Systems, Oxford, UK

Background: To model the financial impact of specimen rejection due to preanalytical errors, using institution specific data.

Materials and methods: As part of BD Laboratory Consulting Services ${ }^{\mathrm{TM}}$ a cost of poor quality model was developed to estimate the opportunity cost associated with poor sample quality. Critical data such as overall budget, number of beds, patients of different types/year, sample rejections/year, as well as qualitative data, eg. probable impact of sample rejection were collected by interviewing 
institution staff. The data were then entered into the model to calculate the possible financial impact of laboratory sample rejections. The model separates patients into different groups according to the likely effects of having a sample rejected, the probability that the rejection would have a low, medium or high impact and the consequences on institution time and resources. The overall consequence, or opportunity cost, was expressed as patient treatment time and financial cost.

Results: The size of the institutions $(\mathrm{N}=10)$ ranged from 326 to 1,200 beds, with total operating costs between $€ 41$ million and $€ 1.1$ billion. The number of blood tests per month was between 37,000 and 458,000 and of these between $0.09 \%$ and $2 \%$ were rejected (mean $0.93 \%)$. The total cost of specimen rejection ranged from $€ 22 \mathrm{~K}$ to $€ 5.9$ Million per annum (mean $€ 1.9$ Million), equating to a percentage of total operating costs from $0.1 \%$ to $1.2 \%$ (mean $0.4 \%$ ). The estimated cost per patient for a sample rejection was from $€ 29$ to $€ 349$ (mean $€ 171$ ).

Conclusions: A customisable model which allows institutions to estimate, using local data and assumptions, the effect of sample rejection on patient treatment and cost. Results can be compared to those from other institutions to benchmark performance and assumptions. The results demonstrated that a reduction in the number of rejected samples due to preanalytical errors could lead to significant cost savings for most institutions.

*Corresponding author: stephen_church@europe.bd.com

\section{P31}

\section{Using BD Laboratory Consulting Services $^{\mathrm{Tm}}$ to understand the impact of the preanalytical phase on sample quality and safety, a multi country perspective}

\author{
Schlueter $K^{* 1}$, Church S2 \\ 1BD Diagnostics, Preanalytical Systems, Heidelberg, Germany \\ ${ }^{2} \mathrm{BD}$ Diagnostics, Preanalytical Systems, Oxford, UK
}

Background: The complexity of the preanalytical (PA) phase has precluded standardisation of PA processes, despite its impact on sample quality, laboratory efficiency, or patient \& healthcare worker safety. The BD Laboratory Consulting Services ${ }^{\mathrm{TM}}$ Preanalytical Review audits PA procedures and practices in hospitals in different countries. Processes were assessed from storage of blood collection materials through specimen collection, transportation, processing of the samples and the resulting sample quality. By following the samples through the complete process, it was possible to link specific PA attributes to sample quality deficiencies.

Materials and methods: A consistent method and data collection form were used for audits ( $\mathrm{N}=$ 48) of all blood collection systems. Data were collected by observation of the PA phase. Sample quality was assessed for laboratory samples of the same type.

Results: The PA phase was observed for 3597 blood collection tubes over 1350 collections. Sample quality was assessed for 8016 chemistry and 3532 coagulation tubes. For collections that resulted in hemolysed samples, $48 \%$ had prolonged use of tourniquet, $31 \%$ used catheters and for $38 \%$ the disinfectant was not allowed to dry. For serum samples with fibrin where the PA process had been observed, $26 \%$ had less than 30 minutes between collection and centrifugation and $81 \%$ had not been mixed. The following list gives the percentage of collections where a particular behaviour was observed, incorrect patient identification procedure, 56\%; tubes labelled prior to collection 61\%; 
coagulation tubes filled to less than $90 \%$ of tube volume $7 \%$; gloves not worn $37 \%$; incorrect activation of needle safety device $19 \%$.

Conclusions: The BD Preanalytical Review standardised audit methodology allows comparison of results between departments and institutions. The prospective nature of the reviews permits identification of issues based on more data than from rejected samples alone and therefore affords a more complete understanding for those involved in the PA phase.

*Corresponding author: kathrin_schlueter@europe.bd.com

\section{P32}

\section{How to mix blood collection tubes, a comparative evaluation of the GME Labsystems T-Swing automated tube mixer vs. manual mixing during phlebotomy}

\section{Ford $K^{*}$, Church S}

BD Diagnostics, Preanalytical Systems, Oxford, UK

Background: Mixing of blood collection tubes is an important process which if not competed correctly has the potential to induce platelet clumping, haemolysis, and other sample artifacts. Recommendations for mixing are poorly adhered to. The GME Labsystems T-Swing is an automated sample mixer. This study evaluated the T-Swing mixer for effects on sample quality \& key analytes.

Materials and methods: The performance of the mixer was compared against recommended manual mixing by the phlebotomist at the time of sample collection. Samples were collected from apparently healthy individuals in the correct order (one discard tube followed by twelve BD Vacutainer ${ }^{\circledR}$ Blood collection tubes, Citrate (3), EDTA (3), BD $\mathrm{SST}^{\mathrm{TM}}$ II (3), BD PST ${ }^{\mathrm{TM}} \boldsymbol{I I}$ (3). One set was mixed by the phlebotomist according to the manufacturer's recommendations and the other two sets using the T-Swing mixer. With one of these sets the tubes were mixed either 4 or 8 times (whichever was closest to Manufacturer's recommendations) and all tubes in the third set were mixed 4 times. The appropriate samples were analysed for routine coagulation (PT \& aPTT), haematology (FBC) and biochemistry tests. The results were compared for each sample set, along with visual observations of the samples.

Results: Equivalent performance was demonstrated for all visual observations and analyte comparisons. No haemolysis was observed in the serum (BD SST $\left.{ }^{\mathrm{TM}} \mathrm{II}\right)$ or plasma (BD PST ${ }^{\mathrm{TM} I I)}$ tubes, analytes biases were for potassium (BD SST ${ }^{\mathrm{TM}} \|<0.5 \%, \mathrm{BD}$ $\mathrm{PST}^{\mathrm{TM}} \|<3.0 \%$ ) and LD (BD SST ${ }^{\mathrm{TM}} \|<1.0 \%$, BD PST $^{\mathrm{TM}} \|$ $<7.0 \%)$. All EDTA tubes showed no platelet clumping (bias $<1.0 \%$ for 4 or 8 mixes) indicating effective mixing.

Conclusions: Equivalent performance was demonstrated between the automated methods and the manual mixing method, with no increase in the incidence of haemolysis or platelet clumping. The data suggest that four automated inversions is equivalent to recommended number of manual inversions.

*Corresponding author: karen_ford@europe.bd.com

\section{P33}

\section{Accuracy evaluation of two blood glucose monitoring systems according to prediction-error grid analysis}

Isgrò MA, Morelli R, Colacicco L, Zuppi C, Scribano D*

Dipartimento di Medicina di Laboratorio, Cattedra di Biochimica Clinica e Biologia Molecolare Clinica, U.O.C. Analisi I, Policlinico "A. Gemelli", Roma, Italia

Background: The accuracy of blood glucose (BG) self monitoring systems is mandatory, as reliable results are prerequisite for therapeutic decisions.

Materials and methods: We evaluated two BG monitoring systems: Menarini StatStrip Xpress and Bayer Breeze 2 for system accuracy according to ISO 15197:2003, AACC/NACB guidelines and Clarke 
error grids. BG measurements of 74 patients were performed on both systems and then compared with results obtained using hexokinase reaction on ROCHE Cobas 8000.

Results: We compared glucose results using t-test, Mann-Whitney test, Passing-Bablok linear regression and Bland-Altman plots. T-test showed a statistical difference $(P<0.03)$ of Menarini BG systems: mean glucose $=126.6 \mathrm{mg} / \mathrm{dL}$ (range 78-301), SD = $37.6 \mathrm{mg} / \mathrm{dL}, \mathrm{CV} \%=29.7$ vs. laboratory values: mean glucose $=112.9 \mathrm{mg} / \mathrm{dL}$ (range 72-295), $\mathrm{SD}=37.5$ $\mathrm{mg} / \mathrm{dL}, \mathrm{CV} \%=33.2$, while it wasn't found any statistical difference $(P<0.12)$ for Bayer BG systems: mean glucose results $=117.4 \mathrm{mg} / \mathrm{dL}$ (range 71-293), $\mathrm{DS}=36.6 \mathrm{mg} / \mathrm{dL}, \mathrm{CV} \%=31.1$. Mann-Whitney test further confirmed the statistical difference $(P<$ 0.003 ) of Menarini BG vs. laboratory values. Passing-Bablok linear regression and Bland-Altman plots showed that both BG monitoring systems fulfilled ISO 15197 requirements (the percentage of results showing the minimum acceptable accuracy was $100.0 \%$ for either StatStrip Xpress or Breeze ${ }^{\circ}$ ). Nevertheless, only Breeze 2 meets the AACC/NACB guidelines, but not StatStrip Xpress (95.9\% vs. $86.5 \%$ of results showed the minimum acceptable accuracy, respectively). According to Clarke error grids, both StatStrip Xpress and Breeze 2 had $100.0 \%$ of their results in error zones $A$ and $B$, considered as "clinically uncritical".

Conclusions Both BG systems fulfilled the minimal accuracy ISO standards' requirements and didn't present clinically critical errors, but only Breeze 2 met the more stringent AACC/NACB guidelines. Because inaccurate systems bear the risk of false therapeutic decisions and subsequent possible severe health injury, regular and standardized evaluation of BG meters and test strips should be performed by manufacturers in order to ensure adherence to more recent guidelines.

Key words: blood glucose monitoring systems; accuracy evaluation; prediction-error grid analysis

*Corresponding author: d.scribano@rm.unicatt.it

\section{P34}

\section{Lipemia as nonconformity of blood donor samples}

\author{
Miletić Lovrić M*, Vuk T, Stojić Vidović M, Mihaljević I \\ Croatian Institute of Transfusion Medicine (CITM), Zagreb, \\ Croatia
}

Background: Mandatory screening of blood donors for transfusion-transmitted diseases in Croatia includes HBsAg, anti-HCV and HCV Ag, HIV Ag/Ab and antibodies to Treponema pallidum (TP). For that purpose we use the most sensitive and specific immunoassays. The part of preanalytical phase of testing is also organoleptic examination of the samples for pronounced lipemia, hemolysis and icterus. According to the most manufactures of serology immunoassays there is potential interference from elevated levels of triglycerides ( $>34$ $\mathrm{mmol} / \mathrm{L}$ ), hemoglobin ( $>5 \mathrm{~g} / \mathrm{L}$ ) and bilirubin ( $>342$ $\mu \mathrm{mol} / \mathrm{L}$ ) and it is usually $10-20 \%$ difference in the test unit (ratio = optical density/cut-off). And also there is possibility of invalid test result. We evaluate these samples as unsuitable or nonconforming due to our guidelines for management of nonconforming samples.

Materials and methods: In the period between January $1^{\text {st }}$ and October 31, 2012 we have collected 84,512 blood donations and so much blood samples we organoleptic examined for lipemia, hemolysis and icterus, and tested for mentioned markers: HBsAg, anti-HCV and HCV Ag, HIV Ag/Ab and anti-TP, used Abbott Prism and Architect tests and Bio-Rad Ultra tests.

Results: 20 of 84,512 samples we evaluated as pronounced lipemic $(0.024 \%)$ and only 1 as pronounced hemolytic $(0.0012 \%)$.

Conclusion: The most common cause of nonconformities in our Institute are lipemic samples, compare to other nonconformities together: incomplete clotting, hemolysis, labeling errors, icterus, inadequate quantity and others. There is notable reduction of samples nonconfirmties, primarily because of reduced frequency of lipemic samples. 
That is due to systemic education of blood donors on the favorable pre-donation dietery habits which has been performed for many years in our Institute.

Key words: nonconformity; lipemic samples; blood donors

*Corresponding author: manuela.lovric@hztm.hr

\section{P35}

\section{Effect of hemolysis on routine coagulation assays}

\author{
Milevoj Kopcinovic L*, Bronic A, Hreljac Sevcenko M, Pavic M \\ University Department of Chemistry, University Hospital of \\ Traumatology, Medical School University Hospital Sestre \\ Milosrdnice, Zagreb, Croatia
}

Background: Hemolysis might affect the reliability of laboratory testing and is reported to be the leading source of unsuitable specimens. Our aim was to investigate the effect of in vitro hemolysis on routine coagulation parameters.

Materials and methods: We studied 50 pairs of hemolyzed and subsequently re-collected nonhemolyzed plasma samples within individual patients. The time interval between the two collections was recorded. Hemolysis and approximate hemoglobin concentration were assessed by visual inspection, comparing samples against a standard color scale. According to hemoglobin concentration, hemolyzed samples were divided into 4 subgroups: slightly $(0.5 \mathrm{~g} / \mathrm{L})$, mildly $(1 \mathrm{~g} / \mathrm{L})$, moderately ( $2 \mathrm{~g} / \mathrm{L})$ and severely (> $3.0 \mathrm{~g} / \mathrm{L})$. PT, APTT and fibrinogen were determined in all samples immediately after collection, using an automated analyzer. Differences between hemolyzed and corresponding non-hemolyzed specimens were evaluated. The effect of hemolysis was expressed as interfering bias and compared to analytical quality specifications and reference change values to define analytically and clinically relevant variations.

Results: Statistically significant overestimation of PT values was observed in all investigated sub- groups. APTT was significantly shortened only in the severely hemolyzed subgroup (23.9 (22.0-27.8) s vs. 26.5 (24.0-29.1) s, $P=0.002$ ). Likewise, fibrinogen concentrations were overestimated in the severe hemolysis subgroup (4.5 (3.7-5.5) $\mathrm{g} / \mathrm{L}$ vs. 4.4 (3.5-5.5) $\mathrm{g} / \mathrm{L}, \mathrm{P}=0.005)$. The mean interfering biases were heterogeneous for all assayed parameters, independent of hemolysis degree. When compared to desirable specifications, investigated parameters showed no analytically or clinically relevant variation.

Conclusions: Although the differences between hemolyzed and non hemolyzed specimens observed in this study were not analytically nor clinically relevant, they were heterogeneous and unpredictable, which emphasizes the need to thoroughly investigate this issue and eventually revise, standardize and harmonize procedures for management of unsuitable samples in coagulation testing.

Key words: hemolysis; coagulation testing; unsuitable specimens

*Corresponding author: laramilevoj@gmail.com

\section{P36}

\section{Design of a high quality and controlled process for a centralized phlebotomy unit}

\section{Kot A, de Jonge Niels*}

Centraal Klinisch Chemisch Laboratorium (CKCL), Leiden University Medical Center, Leiden, The Netherlands

Background: The error rate in the preanalytical phase is still relatively high. Main root causes are an insufficient control mechanism for quality and the presence of waste. In our hospital, phlebotomy used to be a decentralised responsibility, i.e. each ward and outpatient clinic was responsible for the phlebotomy in their patients. Recently, it was decided to bring phlebotomy under the responsibility of the laboratory and to centralise phlebotomy for outpatients in a new facility. Our objective was 
to design a high quality and controlled phlebotomy process. Total turnaround time should be minimal in order to facilitate clinical pathways.

Materials and methods: Our hospital is a medium-sized academic hospital, performing over 130.000 phlebotomy procedures for outpatients (approximately 500-700 patients per day). We applied the Delft Systems Approach operational research to analyse the current situation and to redesign the process. Lean-tools were used to eliminate waste and to increase the productivity. Finally, an adequate capacity was calculated to guarantee a high service level and minimize waiting times, using Kendall's Queueing Theory.

Results: The process redesign for the new phlebotomy process includes a pragmatic model to meet the defined requirements for high patient safety, patient satisfaction and productivity. With the elimination of waste a $30 \%$ reduction of labour can be gained. The calculation of the capacity was validated for one of the current facilities. The capacity was calculated to guarantee a maximal average waiting time of 4 minutes for at least $90 \%$ of the patients. The overall average waiting time is estimated to be 30 seconds and total average turnaround time less than 7 minutes

Conclusions: The Delft Systems Approach is a valuable methodology for both process analysis and redesign. Kendall's Queueing Theory was validated as a tool to calculate the capacity and forecast waiting and service times in a phlebotomy facility.

*Corresponding author: N.de_Jonge@lumc.nl

\section{P37}

\section{A case report of EDTA contamination and high potassium result in non-hemolyzed serum}

\section{Banys V}

Vilnius University Faculty of Medicine, Department of Physiology, Biochemistry, Microbiology and Laboratory Medicine, Vilnius, Lithuania

Background: EDTA contamination of serum samples is one of pre-analytical errors occurring in daily work. EDTA may be present in serum due to improper order of tubes (EDTA tube being drawn before serum tube - possibility of backflow of EDTA into the collection device and carryover of additive into serum tube) or manual transfusion of EDTA blood into serum tube. EDTA causes hypocalcemia, hypomagnesiemia (due to chelation) and subsequently decreased activity of alkaline phosphatase. A case of high potassium value in non-hemolyzed serum due to EDTA contamination is reported.

Materials and methods: 61 years old male presented to hospital with hip fracture, serum potassium $10.44 \mathrm{mmol} / \mathrm{L}$, normal sodium and chloride results, and calcium result below detection limit of the method. Suspecting pre-analytical error two subsequent samples were drawn in 2 and 4 hours. All samples were retested for potassium, sodium and chloride using three different methods: Beckman Coulter Synchron CX9 (USA), Abbott Architect ci8200 (USA), Radiometer ABL800 (Denmark). Total calcium concentration and alkaline phosphatase activity were measured by first two methods. Hospital administration organized audit on work of phlebotomists.

Results: Mean of first sample (elevated potassium) was $10.31 \mathrm{mmol} / \mathrm{L}(\mathrm{SD}=0.27, \mathrm{~N}=3$ ). Means of other samples (normal potassium) were $5.14 \mathrm{mmol} / \mathrm{L}$ $(\mathrm{SD}=0.07, \mathrm{~N}=3)$ and $4.85 \mathrm{mmol} / \mathrm{L}(\mathrm{SD}=0.04, \mathrm{~N}=$ 3) respectively. Alkaline phosphatase activity (58 vs. 87 IU/L Architect; 43 vs. 69 IU/L Synchron) and total calcium concentration (0.63 vs. $1.92 \mathrm{mmol} / \mathrm{L}$ Architect; 1.11 vs. $2.09 \mathrm{mmol} / \mathrm{L}$ Synchron) of first sample was decreased significantly comparing to other 
samples. Sodium and chloride results were uniform and normal. Audit revealed a nurse who manually transferred part of EDTA blood to serum tube.

Conclusions: Irresponsible behavior of nurses may produce erroneous biochemical data, lead to incorrect diagnosis and unnecessary treatment. Education of medical and nursing staff regarding proper blood collection techniques is needed to prevent sample contamination.

Key words: EDTA; potassium; contamination

Corresponding author: valdas.banys@santa.lt

\section{P38}

\section{Performance of aprotinin tubes for routine ACTH assay in the Hospital Unit with manual transport system}

\author{
Pollak J*, Senterkiewicz L, Sypniewska G
}

Department of Laboratory Medicine, Collegium Medicum in Bydgoszcz, Nicolaus Copernicus University in Torun

Background: Preanalytical factors such as proteolytic degradation can affect reliability of hormone assay results. Adrenocorticotropic hormone (ACTH) in blood is considered highly unstable. Manual transport system of the tubes, especially at night, led to discordant ACTH results between night and morning collection. The aim of the study was to investigate the effect of different anticoagulants on the stability of ACTH in test tubes transported with the use of manual transport system.

Materials and methods: Blood was collected from 26 patients twice: at 8 am and 11 pm into 2 different types of EDTA-containing tubes, with or without the presence of aprotinin. Both tubes from each patient were brought on ice to the laboratory within 1 hour, centrifuged and assayed immediately using the Roche-ACTH-electrochemiluminescence immunoassay on fully automated Cobas e411 analyser.

Results: ACTH concentrations were: 1-731 pg/mL in EDTA tubes and 1.61-992 pg/mL in EDTA-aproti- nine tubes taken at $8 \mathrm{am} ; 1-60 \mathrm{pg} / \mathrm{mL}$ in EDTA tubes and $1-101 \mathrm{pg} / \mathrm{mL}$ in EDTA-aprotinin tubes taken at $11 \mathrm{pm}$. Results were compared between two different types of tubes and expressed as mean percentage of difference. Results showed that plasma ACTH concentration in EDTA-aprotinin tubes was always higher than in EDTA-tubes, respectively: $15 \%$ in samples collected at 8 am $(P<$ $0.05)$ and $9.35 \%$ in samples collected at $11 \mathrm{pm}(\mathrm{P}<$ 0.05).

Conclusions: The addition of aprotinin to EDTAtubes showed an improvement of ACTH stability in the blood that is of essential importance in the hospitals without pneumatic tube transport system.

Key words: ACTH; aprotinin; hormone stability

*Corresponding author: asiapollak@wp.pl

\section{P39}

\section{The effect of standardization of sampling procedure to the proportion of haemolytic and clotted samples}

\section{Reimann K*, Osi K}

Central Laboratory, East Tallinn Central Hospital, Tallinn, Estonia

Background: Clotting and haemolysis are the most frequent problems in blood sampling. In the East-Tallinn Central Hospital (ETCH), standardization of sampling procedure was carried out in December 2009 in attempt to reduce the number of unusable samples. New sampling sets were implemented and training for the whole hospital nursing staff was performed. Current overview evaluates the effect of these standardization measures on the proportion of clotted and haemolytic samples.

Materials and methods: Proportion of samples, considered clotted or haemolytic by visual inspection, was calculated for hospital's surgery, internal diseases', and women's clinics in 2009, 2010 and 2011. Statistical significance of the yearly differences was assessed using Chi-square test. 
Results: The total proportion of clotted samples decreased from $0.04 \%$ in 2009 to $0.03 \%$ in 2010 (P $=0.004)$; and this reduction was maintained in 2011. The total proportion of haemolytic samples was reduced from 2009 to 2010 (respectively 0.19 and $0.11 \%, P<0.001)$; the reduction continued in 2011, when the proportion of haemolytic samples was $0.09 \%$. Regarding yearly changes in the clinics, the proportion of clotted and haemolytic samples decreased statistically significantly in all clinics between 2009 and 2010 with the exception of clotted samples in women's clinic where the reduction was achieved only in 2011. In women's and internal diseases' clinics the reduction in proportion of haemolytic samples continued in 2011. The reduction, achieved in 2010 in proportions of haemolytic samples in surgery clinic, was maintained in 2011. The proportion of clotted samples in surgery and internal diseases' clinics in 2011, however, returned to the level of 2009.

Conclusions: The standardization of blood sampling in the ETCH was associated with reduction in proportions in both clotted and haemolytic samples. Yet for maintaining the reduction, repeated staff trainings may be necessary.

Key words: blood sampling; standardization; clotting; haemolysis

*Corresponding author: kristiina.reimann@itk.ee

\section{P40}

\section{Preparing the patient as an imperative for obtaining adequate sample for qualitative urinalysis}

\author{
Sikirica $M^{*}$, Radišić Biljak V, Flegar-Meštrić Z \\ Department of Medical Biochemistry and Laboratory Medicine, \\ University Hospital Merkur, Zagreb, Croatia
}

Background: Urine is an analytical matrix that provides information on numerous physiological processes. It is accessible and it can be collected noninvasively what is of great importance. Urinalysis is one of the most frequently requested analy- ses at all levels of health care. Providing the adequate preanalytical conditions for obtaining an appropriate sample is the basic precondition for reliable results and appropriate clinical interpretation afterwards. It can be obtained in a form of oral or written recommendations.

Materials and methods: Anonymous survey in a form of questionnaire was conducted in 78 randomly selected patients who were instructed to the Department of Medical Biochemistry and Laboratory Medicine for laboratory analysis. Our aim was to gain insight into the awareness of patients regarding preanalytical conditions affecting urine sampling. The main question was whether patients received any kind (oral or written) of recommendations how to prepare for giving urine sample either form the physician, nurse or laboratory staff.

Results: Only $13 / 78$ patients received partial verbal instructions ( 5 from the doctor, 8 from the nurse) prior urine sampling, while $65 / 78$ patients had never received any form of recommendations for administering the single sample of urine in the practice of primary care. However, almost $80 \%$ of selected patients (59/78) received written and/or oral instructions when they arrived in their primary laboratory.

Conclusion: These results indicate that patients are poorly instructed for urine sampling in their primary care office, while these instructions are available in most primary laboratories. Informing patients is one of the basic conditions of obtaining a good sample. International recommendations a continuous education of health teams leads to overall reduction of preanalytical errors. The effectiveness of these basic conditions is defined by the quality indicators that objectify all steps in the collection, transport and storage of samples.

*Corresponding author: mirjana.sikirica@hi.htnet.hr 
P41

\section{The preanalytical phase starts before blood collection}

\author{
Attard R*, Farrugia R, Bezzina Wettinger S \\ Department of Applied Biomedical Science, Faculty of Health \\ Sciences, University of Malta Msida, Malta
}

Background: Large biological collections are being set up for biobanking, epidemiological studies and in the search of biomarkers. A number of variables could play an important role in determining levels of analytes in blood even before blood is collected and can result in contradictory outcomes of studies, particularly when levels are compared between groups of individuals. There is a lack of guidelines and standardised instructions that need to be followed prior to phlebotomy.

Materials and methods: A literature search was conducted to determine the variables that can influence results prior to blood collection in a research setting and to draft instructions for participants to follow in the week preceding blood collection.

Results: In addition to fasting, several variables are reported to influence levels of analytes in blood. Depending on the scope of the collection it may be necessary to adopt measures to limit the effect of these variables. These may include: (a) simple recording of the variable (e.g. use of medications, day of menstrual cycle, pregnancy, time and date of phlebotomy since season may also influence variables, time of last food intake, perceived psychological stress levels); (b) postponement of blood draw (e.g. for medical and dental interventions, infection and vaccination, minor injuries in the previous week, blood transfusions and donation); (c) giving instructions to the research subject (e.g. to fast, avoid alcohol, tea, coffee and milk, avoid smoking 12 hours before blood draw and if not possible record time of last cigarette, maintain normal physical activity and dietary habits, avoid strenuous exercise, avoid use of chewing gum before phlebotomy, determine instructions on use of vitamins and supplements, drink water to avoid dehydration, empty bladder before blood draw). Clear explanations which may include a leaflet must be given to the research subject.

Conclusion: There are several factors influencing blood analytes even prior to blood draw and they need to be given considerable attention.

Key words: instructions; prior to blood draw; research; biobanking

*Corresponding author: ritienne_attard@hotmail.com

\section{P42}

\section{The analysis of performance by medical nurses of blood sample collection procedure for laboratory tests}

\author{
Kovalevskaya SN*, Khorovskaya LA, Petrova NG
}

State Medical University n. a. I. P. Pavlov, St.-Petersburg, Russian Federation

Background: Three distinct steps can be identified in the measurement procedure: preanalytical, analytical and postanalytical. It has been reported that a majority of errors (60-70\%) occurs during the preanalytical step, which is heavily depending on human factors. Phlebotomy is a most important part of the preanalytical step. In the Russian Federation blood samples are collected by medical nurses employed by clinical divisions of the Health Care System. The aim of the present study was to analyze skills and knowledge of the nurses.

Materials and methods: A questionnaire comprising 24 questions was developed and distributed among 153 nurses in State and private outpatient clinics and hospitals in 3 cities in different regions of the Russian Federation; St.-Petersburg (North-West Region), Khanty-Mansiysk and Noyabrsk (Siberia). The questionnaire addressed the demographics of respondents, their professional experience and particularly phlebotomy training using evacuated blood collection systems (EBCS), dealing with difficulties encountered during phlebotomy, transport/transfer of blood samples and safety issues/information support. Answers were 
de-identified and then evaluated using general statistical methods.

Results: The investigation revealed that about $20 \%$ of nursing staff was not trained in EBCS systems. Of those that were trained $55 \%$ attended courses given by manufactures of EBCS and $45 \%$ were trained by their colleagues. Half of respondents (50\%) had difficulties to answer to the questions concerning method for obtaining samples with EBCS. $86 \%$ of phlebotomists answered questions about rules for application of a tourniquet incorrectly and $75 \%$ were unaware of the volumes of blood required for different types of evacuated tubes. More than $50 \%$ of the respondents reported difficulties during venipuncture. Most commonly are: causes to recollect of sample collection (80\%), difficulties to locate a vein (40\%), hazard of injury by used or unused equipment $(28 \%)$, venipuncture of neonates and children less than one year old (10\%) were encountered. The most frequent reasons for duplication of sample collection were: hemolysis of samples (80\%), errors in sample labeling (16\%) and broken evacuated tubes (4\%). The survey revealed that $7 \%$ of nurses refused to use EBCS and preferred to work with traditional methods.

Conclusion: The study indicates that there is an insufficient training of phlebotomists in using EBCS. This creates a potential risk for patients and needs attention in formulating the curriculum for medical nurses within the Russian Federation Health Care System.

Key words: phlebotomy; preanalytical stage; medical nurses

*Corresponding author: kovalevskaya@labstory.ru
P43

\section{Frequencies of hemolysis and lipemia in whole blood samples in pediatric population}

\author{
Grzunov A*, Jagetic R, Lenicek Krleza J \\ Children's Hospital Zagreb, Department of Laboratory \\ Diagnostics, Zagreb, Croatia
}

Introduction: The aim of this study was to assess prevalence of interference from hemolysis and lipemia in whole blood samples in pediatric population.

Materials and methods: 3903 consecutive samples collected during one month period were visually monitored for turbidity and hemolysis and patients age were noted. The statistical significance of differences between relative frequencies of interferences was calculated with $x^{2}$ test.

Results: $7 \%$ of all samples were identified to have hemolysis. Hemolysis in samples drawn from vein occurred more frequently than hemolysis in capillary samples $(\mathrm{P}<0.001) .18 \%$ of samples were identified to have lipemia. Both lipemia and hemolysis were observed in $<1 \%$ of samples. Interferences were more frequent in population up to 2 years old $(P<0.001)$.

Conclusion: Due to presence of interferences in significant number of samples, particularly in population up to 2 years old, a considerable number of whole blood samples may be unreliable. Continuous education and training of healthcare personnel in phlebotomy and patient preparation prior to sample collection is needed.

Key words: whole blood sample; hemolysis; lipemia; interference; child

*Corresponding author: anagrzunov@gmail.com 
P44

\section{National guideline for phlebotomy in the Netherlands}

Eppens $\mathrm{E}^{1}$, Roelofs-Thijssen $\mathrm{M}^{2}$, de Jonge $\mathrm{N}^{3}$, van Dongen-Lases $\mathrm{E}^{* 4}$

'Department of Clinical Chemistry, Gelderse Vallei Hospital, Ede, the Netherlands

2Department of Clinical Chemistry, Rijnstate Hospital, Arnhem, the Netherlands

3Department of Clinical Chemistry, Leiden University Medical Center, Leiden, the Netherlands

${ }^{4}$ Department of Clinical Chemistry, Academic Medical Center, Amsterdam, the Netherlands

Background: The Working Group Preanalytical Phase of the Netherlands Society for Clinical Chemistry and Laboratory Medicine addresses all elements of the preanalytical phase of clinical chemistry laboratory testing in the Netherlands. One of its major tasks is to develop national guidelines for the preanalytical phase. Therefore, the Dutch Working Group Preanalytical Phase recently developed the national guideline for phlebotomy. The aim of this guideline was to summarize the best practices in phlebotomy in order to improve the safety of patients and phlebotomists and the quality of laboratory testing.

Materials and methods: Information on phlebotomy was collected and summarized by the Dutch Working Group Preanalytical Phase. Information was gathered from many resources such as national legislation e.g. the Individual Healthcare Professions Act, national guidelines e.g. guidelines concerning hygiene from the Dutch Working Group Infection Prevention, international guidelines e.g. guidelines from the Clinical and Laboratory Standards Institute and literature. In April 2013, the national guideline for phlebotomy will be approved by the Netherlands Society for Clinical Chemistry and Laboratory Medicine.

Results: The national guideline for phlebotomy in the Netherlands has been divided in chapters and subdivided in sections, each presenting background information on the specific aspects of phlebotomy, e.g. factors that affect laboratory values, facilities, supplies, venepuncture procedure, venepuncture in children and difficult collections, competence of phlebotomists, hygiene and safety. Each section ends with minimum standards and/ or recommendations.

Conclusions: The national guideline for phlebotomy in the Netherlands describes the best practices in phlebotomy. Compliance with this guideline will improve the safety of patients and phlebotomists and the quality of laboratory testing.

Key words: guideline; national; phlebotomy

*Corresponding author: e.c.vandongen-lases@amc.nl

\section{P45}

\section{Reference ranges for coagulation screening tests and coagulation inhibitors in newborn's cord blood on ACL TOP 500}

Trampuš Bakija $A^{* 1}$, Benedik Dolničar $\mathrm{M}^{2}$, Mencin $\mathrm{D}^{1}$, Prelec $\mathrm{A}^{3}$, Kitanovski L ${ }^{2}$

'UMC Ljubljana, University Children's Hospital, Unit of Special Laboratory Diagnostics, Ljubljana, Slovenia

2UMC Ljubljana, University Children's Hospital, Department of Oncology and Hematology, Ljubljana, Slovenia

${ }^{3}$ UMC Ljubljana, Division of Gynecology and Obstetric, Department of Perinatology, Ljubljana, Slovenia

Background: The concentration and function of coagulation factors and natural inhibitors depend on gestational and postnatal age of the infant. The concept of developmental haemostasis is broadly accepted and age related reference values should be concerned in interpretation of laboratory results. It is also recommended that absolute values of reference ranges for coagulation assays should be established for reagent/analyzer system. Our study was designed to determine reference ranges for newborns for activated partial thromboplastin time (APTT), prothrombin time (PT), thrombin time (TT), fibrinogen, antithrombin (AT), protein C activity and free protein $\mathrm{S}$. 
Materials and methods: Plasma samples were obtained from umbilical vein from 50 healthy full term neonates born in University Medical Centre Ljubljana. All tests were performed on ACL TOP 500 coagulation analyzer (Instrumentation Laboratory) using reagents from Instrumentation Laboratory. Reference ranges and t-test for statistical differences were calculated using MedCalc software.

Results: Reference values for tests are: APTT: $32 \mathrm{~s}$ (23-41), PT [s]: 12.2 s (10.0-14.3), PT [\%]: 87\% (62109), TT: $18 \mathrm{~s}$ (15-21), fibrinogen: $1.72 \mathrm{~g} / \mathrm{L}(1.1-2.4)$, AT: $61 \%$ (37-85), protein C activity: 36\% (20-52) and free protein S: 49\% (28-71). Values for all tests are significantly different $(P<0.001)$ from those for adults or manufacturer. Compared to scarce published literature, the directions of age-related changes are consistent, but the magnitude is specific for reagent/analyzer combination.

Conclusions: Our data confirm previously reported influence of lower thrombin generation potential and age related changes of inhibitors in neonates that results in prolongation of screening tests and marked reduction of inhibitor activity. Adult reference ranges for coagulation screening tests, especially PT and APTT and inhibitor activity tests cannot be applied to newborns. Age related test specific reference values are needed for accurate diagnosis and management of children with suspected bleeding disorder.

*Corresponding author: alenka.trampus@guest.arnes.si

\section{P46}

\section{Troponin T high sensitivity assay: serum or lithium heparin as specimen type?}

\section{Mulliez S, Stove V, Delanghe J*}

Department of Clinical chemistry, microbiology and immunology, Ghent University Hospital, Belgium

Background: The manufacturer of the TroponinT high sensitivity (TnT hs) assay states in its reagent leaflet that serum or $\mathrm{K}_{2}$-EDTA, Li-heparin and Na- heparin plasma are acceptable specimen types for analysis. However, heparin has been described to bind some isoforms of TroponinT, especially the forms distributed in circulation in early phases of myocardial injury. Our aim was to further evaluate the influence of the specimen type in particular in patients with acute myocardial infarction (AMI).

Materials and methods: A total of 33 paired samples were drawn in parallel from patients suspected with AMI on their admission at the Ghent University Hospital. Venoject serum separator (VF054SAS) and Li-heparin (VF054SHL) tubes were used (Terumo). cTnT was measured by the Elecsys hsTnT assay (Roche Diagnostics). Analysis occurred within 1 hour after sample collection. All patients gave informed consent. To exclude potential interference from hemolysis, samples with values of $\mathrm{H}$-index $\geq 88$ were not used in further calculations.

Results: No statistically significant differences were found between specimen types ( $P>0.05$ ). However, in all patients with the final diagnosis of AMI $(\mathrm{N}=7)$, CTnT concentrations in heparin plasma were found to be lower than those measured in serum (mean difference of $-11.4 \%$, range -1.2 to $-19.8 \%)$. We also observed a large variability in the plasmaTnT/serumTnT ratio among the individual sample pairs of these AMI patients. The magnitude of the difference between serum and plasma was independent of the absolute concentration.

Conclusions: Our data show that serum and heparin plasma samples are not suited to be used interchangeably for AMI patients. The concentration of troponinT measured in heparin-plasma was markedly lower than in serum in AMI patients. Therefore, we have informed our clinicians to stick with one sample types for troponinT analysis, i.e. in our hospital only serum samples are accepted.

Key words: cardiac Troponin T; specimen types; Elecsys high sensitivity assay

\footnotetext{
*Corresponding author: joris.delanghe@ugent.be
} 
P47

\section{Transportation of blood samples through adjacent phlebotomist cabins to the laboratory by a simple conveyer belt reduces turn around time for the samples and waiting time for the patients and may make recommended hand inversions of samples with additives superfluous}

\author{
Stender S*, Mandir IA \\ Department of Clinical Biochemistry, Copenhagen University \\ Hospital, Gentofte, Denmark
}

Background: In our out-patient clinic blood samples are drawn by phlebotomists in 5 separate but adjacent cabins placed as an elongation of the laboratory. In order to reduce turn around time (TAT) we installed a conveyer belt and the phlebotomists placed the samples on the belt during or immediately after each phlebotomy ( 4500/month).

Materials and methods: The belt runs $18 \mathrm{~m}$ with a constant speed $(15 \mathrm{~cm} / \mathrm{second})$ below the windowsill through all 5 cabins and into the laboratory and delivers the blood samples close to the centrifugation unit. During passage of a fire wall the samples are slightly tilted and for the last $6 \mathrm{~m}$ within the laboratory, the belt has a slight upwards slope. The samples make a 180 degree inversion when they leave the belt and end in a tray.

Results: We observed a reduction from 17 to 14 minutes from the hematology samples were drawn until the results were available, when comparing the median values obtained for months before with values obtained for months after installation of the belt. We also observed a reduction from 15 to 10 minutes in median waiting time for the patients from they arrived to the out-patient clinic until they entered the phlebotomist cabins. In contrast to the procedures before the conveyer belt, now the phlebotomists do not have to leave the cabins except for their planned intermissions. We found the same number of thrombocytes in EDTAcoated tubes with blood drawn from 507 consecu- tive patients placed on the belt without any inversions compared to samples drawn from the same venipuncture in similar tubes, inverted 8-10 times as recommended in various guidelines before they were placed on the belt.

Conclusion: The conveyer belt in our setting reduces TAT, increases the number of phlebotomies / hour / phlebotomist and may make recommended inversions of blood tubes with additives superfluous.

Key words: conveyer belt; phlebotomy; TAT; inversion of blood tubes

*Corresponding author: steen.stender@regionh.dk

\section{P48}

\section{Blood samples with additives. Stirred - not shaken. Is that enough for sufficient mixing?}

\section{Stender S}

Department of Clinical Biochemistry, Copenhagen University Hospital, Gentofte, Denmark

Background: Guidelines recommend gentle mixing i.e. 4-10 times inversions of blood samples with additives immediately after phlebotomy. We wanted to find the minimum number of inversions that ensures a sufficient mixing of the blood with the additives.

Materials and methods: Two samples of blood were drawn in 2 identical EDTA-tubes from the same venipuncture from consecutive patients visiting the hospitals out-patient clinic for phlebotomy. One EDTA-tube was inverted 8-10 times as recommended in the guidelines immediately after the venipuncture, whereas the experimental tube was gently placed in a rack in room temperature and remained there for at least half an hour. Both EDTA-tubes were analyzed on the same equipment. The same type of experiments was conducted with samples collected from bedridden patients by phlebotomists at their rounds in the vari- 
ous clinical departments. In the first experiment the experimental tubes received only the mixing that occurred during the walking from the bed to the laboratory. In the second experiment the experimental tubes were inverted only once immediately after phlebotomy. Based on double analysis of the number of thrombocytes in the same EDTA-tube, a more than $20 \%$ drop in thenumber of thrombocytes in the experimental EDTA-tube, was taken as a sign of coagulation due to insufficient mixing of blood and EDTA.

Results: Based on 604 EDTA-tubes placed in a rack immediately after phlebotomy without any inversions for half an hour, $2.8 \%$ were insufficiently mixed. Based on 1009 EDTA-tubes without any planned inversions after phlebotomy of bedridden patients, $0.6 \%$ was insufficiently mixed, while 1032 EDTA-tubes inverted only once, a maximum of $0.4 \%$ were insufficiently mixed.

Conclusion: Insufficient mixing of blood and EDTA in an EDTA-tube is nowadays a rare event even without any inversion of the tube. Guidelines presently recommend up to 10 inversions although fewer may be sufficient.

Key words: guidelines; EDTA-tubes; inversion of blood tubes; phlebotomy; sufficient mixing

Corresponding author: steen.stender@regionh.dk

\section{P49}

\section{Risk of hemolysis in the Emergency Department depending on phlebotomy practices}

\author{
Prieto Alvarez N*1, Gómez-Rioja R², Alcaide Martín MJ2, Barba \\ Yegro MR', Fernandez-Calle P2 , Torres Hidalgo $A^{1}$ \\ 'Emergency Department, La Paz University Hospital, Madrid, \\ Spain \\ 2Emergency Laboratory, La Paz University Hospital, Madrid, \\ Spain
}

Background: In the University Hospital of "La Paz" Emergency Department, the prevalence of hemolyzed specimens is estimated around $14.4 \%$. This rate of hemolysis is higher as compared with other services and can be related to phlebotomy practices, mainly because of the frequent use of catheter. The objective of this study was to analyze the relation between current practices of phlebotomy in our department and the quality of the samples.

Materials and methods: For one month, six nurses from the Emergency department registered the collection procedures used in every patients, categorized as: straight needle + vacutainer, butterfly needle + vacutainer, straight needle + syringe, catheter and arterial blood collection instead of phlebotomy. Hemolysis was quantified as serum index in a Dimension Vista (Siemens HD) and considered as significant by the laboratory.

Results: 435 samples were included. Catheterdrawn was the most frequently used $(55 \%$ of patients), followed by Butterfly needle (20\%), straight needle + vacutainer (13\%), straight needle + syringe (10\%) and arterial-drawn (2\%). Significant differences were observed in the prevalence of hemolyzed samples depending on phlebotomy practice. $50 \%$ of the arterial samples, $30.2 \%$ of syringe-drawn samples, $29.5 \%$ of the catheter-drawn samples, $12.6 \%$ of butterfly needle and $10.3 \%$ of the straight needle + vacutainer were severely hemolyzed. Risk of hemolisys compared to straight needle + vacutainer drawn was calculated using logistic regression. No significant differences were observed between the straight needle + vacutainer and butterfly needle + vacutainer drawn. In the other hand, the use of catheter, syringe or arterial drawn imply an increasing risk (Odds ratio) of 3.6, 3.7 and 8.6 respectively $(P<0.05)$.

Conclusions: We find a clear relation between hemolysis and phlebotomy practices. Catheterdrawn or syringe use, involving blood transfer to the tubes, suppose a 3.7-fold increase in the risk of hemolysis. These practices are used in $65 \%$ of patients in our Emergency Department. To avoid the high prevalence of hemolysis in the Service, it would be necessary to change phlebotomy practices.

Key words: hemolysis; emergency medicine; phlebotomy; catheter

*Corresponding author: noeprieto_22@hotmail.com 
P50

\section{Implementing safe sharps practices in Europe, what do I need to do?}

\author{
De Carli G*1, Abiteboul D², Bouvet E2 , De Schryver A³, Mazón \\ Cuadrado $\mathrm{L}^{4}$, Ncube $\mathrm{F}^{5}$, Puro $\mathrm{V}^{1}$, Wittmann $\mathrm{A}^{6}$, Ippolito $\mathrm{G}^{1}$ for the \\ Sharps Safety in the European Union Group

\footnotetext{
'SIROH, National Institute for Infectious Diseases L. Spallanzani, Rome, Italy

${ }^{2}$ GERES, Faculté de Médecine $\mathrm{X}, \mathrm{BICHAT}$, Paris, France

${ }^{3}$ IDEWE-Universiteit Antwerpen, Leuven, Belgium

${ }^{4}$ Servicio De Prevención Hospital De Fuenlabrada, Madrid, Spain

${ }^{5}$ Bloodborne Viruses Section, National Infectious Disease Surveillance Centre, Health Protection Agency, London, UK ${ }^{6}$ Technischer Infektionsschutz, Bergische Universitaet Wuppertal, Wuppertal, Germany
}

Background: The Directive 2010/32/EU "Prevention from sharp injuries in the hospital and healthcare sector" was issued to protect healthcare workers from the risk of needlestick/sharps injuries and subsequent infection with bloodborne pathogens, and should be adopted within May 2013.

Materials and methods: The Sharps Safety in the European Union Group gathered experts to develop a consensus statement and practical recommendations on the main preventive issues included in the Directive to help in this transition.

Results: Consensus statement: Raise awareness among workers to create a 'need for safety' and overcome resistance to change procedures and devices. Implement an active surveillance of needlestick/sharps injuries and provide feedback to workers. Incorporate Standard Precautions and Safe Injection Practices within education to be delivered at employment and regularly thereafter, particularly when changing technologies or procedures. Link prevention of needlestick/sharps injuries and bloodborne infections through a safe and appropriate use of sharps/needle devices to prevention of healthcare associated infections, to be included in national health plans. Explain rationale and procedures for pre- and post-exposure management, including Hepatitis B vaccination and antiretroviral prophylaxis, which must be al- ways available. Organize work to decrease use of unnecessary needles: concentrate blood tests, switch parenteral therapies to oral as soon as safe for patients, re-evaluate the need for peripheral catheters daily. Place sharps containers where procedures are performed; monitor their use and contents to discourage recapping. Perform task analysis for every procedure involving sharps, especially in surgery. Prioritize procedures involving access to vein/artery in implementing safety-engineered devices incorporating protection mechanisms, followed by all other instances in which an injury may occur regardless of the setting.

Conclusions: Experts must have a proactive role in disseminating the contents and suggesting the best approach to implement preventive measures in Directive 2010/32/EU, helping employers and workers to optimize use of resources.

Key words: prevention; bloodborne infections; occupational injury

*Corresponding author: gabriella.decarli@inmi.it

\section{P51}

\section{Handling of pseudohyponatremia in the routine laboratory workflow}

Siska A*, Seres E, Nagy A

University of Szeged, Department of Laboratory Medicine, Szeged, Hungary

Background: When sodium is measured by some laboratory methods falsely low sodium values can be measured in cases of extreme hyperlipidemia or hyperproteinemia. This phenomenon is called pseudohyponatremia. Our aim was to determine the degree of the lipemia and hyperproteinemia where the direct potetiometry provides more adequate sodium concentration than the indirect one.

Materials and methods: The direct potentiometry equipment was an AVL 9180 and the indirect one was a Roche Modular ISE 900. The lipemia index is automatically measured in our laboratory due to the total laboratory automation. 
Results: In the examined range of both lipemia index and triglyceride concentration there was no significant difference between the sodium levels, measured by the above mentioned methods. However, a weak relationship could be observed between triglyceride concentration and lipemia index in patient samples. In the case of hyperproteinemia significant difference was found between sodium concentrations obtained by the two types of electrode measurement. Above $100 \mathrm{~g} / \mathrm{L}$ of total protein level the sodium measurement without sample dilution (i.e. direct potentiometry) provided clinically more relevant result than the methods with sample dilution (indirect potentiometry).

Conclusion: In accordance with these results the value of sodium in samples with lipemia index below 2000 is acceptable. Above this value a comment should be attached about the possibility of pseudohyponatremia in the laboratory findings. In case of total protein level higher than $100 \mathrm{~g} / \mathrm{L}$ the sodium concentration should be determined by direct potentiometry and the clinicians have to be notified about the used methodology.

Key words: pseudohyponatremia; lipemia; hyperproteinemia; potentiometry

*Corresponding author: siska.andrea@med.u-szeged.hu

\section{P52}

\section{Case report: HAMA interference in determinating CEA concentration in non-Hodgkin's lymphoma patient}

\author{
Stancin N ${ }^{1}$, Derek L ${ }^{* 2}$, Unic A2 ${ }^{2}$ Romic $Z^{2}$, Banek T1 , Ajdukovic \\ Stojisavljevic $R^{3}$ \\ ${ }^{1}$ Center of Nuclear Medicine, Dubrava University Hospital, \\ Zagreb, Croatia \\ ${ }^{2}$ Clinical Department for Laboratory Diagnostics, Dubrava \\ University Hospital, Zagreb, Croatia \\ ${ }^{3}$ Department of Hematology, Dubrava University Hospital, \\ Zagreb, Croatia
}

Background: A 47 year old female was treated for non-Hodgkin's lymphoma CS IV B/B-LCL with immunochemotherapy (R-EPOCH protocol) from
2008-2009. After completing the treatments, follow-up exams included the determination of carcinoembryonic antigen (CEA) concentration. From 2009-2010 CEA concentration repeatedly increased, but using imaging techniques (PET-CT), relapse of the disease was not detected. Due to the suspicion of the possible human anti-mouse antibodies (HAMA) interference, CEA was determined using different immunoassay.

Materials and methods: CEA was determined using chemiluminiscence assay on Vitros ECiQ (Ortho Clinical Diagnostics, Johnson and Johnson, Buckinghamshire, UK), and electrochemiluminiscence assay on Cobas e411 (Hitachi High Technologies Corporation, Tokyo, Japan). Besides the retesting of CEA on different analyzers, dilution test was also performed.

Results: Patient CEA concentrations, determined on Vitros ECiQ vs. Cobas e411 were as follows (3.5 vs. $0.9 \mu \mathrm{g} / \mathrm{L}$ on May 4, 2009; $43.0 \mu \mathrm{g} / \mathrm{L}$ vs. $0.2 \mu \mathrm{g} / \mathrm{L}$ on November 18, 2009; $47.4 \mu \mathrm{g} / \mathrm{L}$ vs. $0.9 \mu \mathrm{g} / \mathrm{L}$ on November 23, 2009 and $60.2 \mu \mathrm{g} / \mathrm{L}$ vs. $1.1 \mu \mathrm{g} / \mathrm{L}$ on May $24,2010)$. Dilution test with 1:2, 1:5 and 1:10 sample dilutions was also performed on Vitros $E C i Q$, and CEA concentrations were not linear $(20.6 \mu \mathrm{g} / \mathrm{L}, 6.3$ $\mu \mathrm{g} / \mathrm{L}$ and $0.5 \mu \mathrm{g} / \mathrm{L}$ respectively).

Conclusions: Increased CEA concentrations on Vitros EciQ that were not in accordance with the clinical status, the deviation from linearity in the dilution test, as well as a significant difference in the concentration of CEA on Cobas e411, point to HAMA interference that was probably a consequence of a immunochemotherapy. Noncompetitive methods that use murine antibodies are most susceptible to interference because of the mice immunoglobulins commonly used in diagnostic and therapeutic procedures. Cases like this can result in a misdiagnosis, errors in patient treatment and monitoring, as well as unnecessary additional testings, so it is necessary be aware of the possible interferences, and collaborate with clinicians so that the diagnostic and clinical disagreement could be clarified as soon as possible.

Key words: human anti-mouse antibodies; carcinoembryonic antigen; immunoassay

*Corresponding author: Iderek@kbd.hr 
P53

\section{Appropriate time of thrombophilia testing related to oral anticoagulant therapy: are recommended guidelines followed}

\author{
Margetić S*, Getaldić B, Ćelap I, Vrkić N \\ Clinical Institute of Chemistry, Medical School Clinical Hospital \\ Center Sestre Milosrdnice, Zagreb, Croatia
}

Background: The appropriate time of testing related to oral anticoagulant therapy (OAT) is one of the crucial preanalytical variables for thrombophilia investigation since many of these tests are affected by this therapy. In order to gain insight into physicians ordering practices, we performed a retrospective analysis of consecutive thrombophilia tests ordered during the 10-month period.

Materials and methods: The study included lupus anticoagulant (LA) investigation and functional assays for protein $C(P C)$, protein (PS) and resistance to activated protein $C$ (APCR), using commercially available coagulation methods (Siemens, Germany).

Results: A total number of tests performed was as follows: 853 (LA), 608 (PC), 443 (PS) and 540 (APCR.) Proportions of positive results for each test were: $\mathrm{LA}=35 / 853$ (4.1\%), $\mathrm{PC}=28 / 608$ (4.6\%), $\mathrm{PS}=50 / 443$ (11.3\%) and APCR $=52 / 540$ (9.6\%). Of all positive test results, 10/35 (28.6\%) for LA, 20/28 (71.4\%) for PC, $22 / 50$ (44\%) for PS and 3/52 (5.8\%) for APCR were attributable to OAT, thus representing falsepositive results that could have considerable implications for patients.

Conclusions: Our findings suggest the appropriate time of testing related to OAT to be simply overlooked by clinicians managing patients with thrombosis, thus resulting with a huge waste of resources with concomitant high likelihood of falsepositive test results. Our findings indicate that thrombophilia testing in patients on OAT still occurs frequently in clinical practice despite recommended guidelines. The net effect of such testing is likely to be more detrimental than beneficial for the patient. On the basis of our results, it is obvi- ous that laboratories need to take a more substantial role in the thrombophilia investigation process than just providing doctors with numbers. The corrective actions should include ongoing education of clinicians and reject testing in patients on OAT since it would be more rational, cost-effective and beneficial for patients.

Key words: thrombophilia testing, oral anticoagulant therapy, preanalytical variable

*Corresponding author: sandra.margetic1@zg.t-com.hr

\section{P54}

\section{Application of Sample Reader inSIGHT2 device for automated reading of barcoded samples at sample reception unit}

Fuček M*, Alpeza Viman I, Zekušić M, Herceg A, Sertić J

Department of Laboratory Diagnostics, University Hospital Centre Zagreb, Croatia

Background: Systemic control of entire laboratory process and monitoring and management of nonconformities are mandatory procedures for every medical laboratory with established quality control system. Along with the most common nonconformities related to sample quality (hemolysis, clot...), a significant number of the nonconformity entitled "sample not delivered" was recorded at Department of Laboratory Diagnostics in 2011. The number of such cases was considerably reduced after introduction of Sample Reader inSIGHT2 device for automated reading of barcoded samples.

Materials and methods: Prior to introduction of Sample Reader inSIGHT2 device, there was no possibility for individual receipt of requests for laboratory management in the laboratory information system (LIS), regardless of whether all samples associated with an individual request have been delivered to the laboratory. The Sample reader inSIGHT2 accepts requests only for the samples delivered to the laboratory and barcoded with the label generated from the hospital information sys- 
tem (HIS) or LIS. Communication with the user takes place via a touch sensitive screen. The device informs the user if a sample has already been received and recorded in LIS and if it is acceptable with regard to the sampling time. The files with accreditation-related data (sample receipt time, identification of the user who has received a sample, identification numbers of received samples) are kept for each received sample while, for the purpose of subsequent forensic management, each sample or test-tube rack is photographed.

Results: After application of Sample Reader inSIGHT2, the nonconformity "sample not delivered" was recorded in 154 cases, as compared to 1092 cases in 2011 (for the periods of three months), while the average receipt time has been reduced significantly.

Conclusion: The application of the device for automated reading of barcoded samples at sample receipt unit of the Department of Laboratory Diagnostics, has allowed rapid and accurate reading of barcoded samples and also a reduced number of the cases of nonconformity entitled "sample not delivered".

Key words: automated reading of barcoded samples; nonconformities

*Corresponding author: mfucek@kbc-zagreb.hr

\section{P55}

\section{Acceptance limits for human blood sample stability: a review}

Oddoze $\mathrm{C}^{1}$, Portugal $\mathrm{H}^{1}$, Avelland $\mathrm{T}^{2}$, Gianolli $\mathrm{JM}^{3}$, Letondel $\mathrm{M}^{4}$, Lalaurie $E^{4}$, Coauch $1^{4}$. GEFAP: Groupe d'Etude Français

d'Amélioration de la phase Préanalytique.

1UTH Timone, Marseille, France

${ }^{2}$ LABM Analys, Marignane, France

${ }^{3}$ LABM des Charpennes, Villeurbanne, France

${ }^{4}$ BD PAS, Pont-de-Claix, France

Background: The preanalytical phase accounts for up to $70 \%$ of the errors occurring in laboratory medicine. Some of these errors occur due to instability of the analyte in the blood matrix which is not only linked to degradation of the sample, but also to external environmental factors such as time delays to sample processing and temperature fluctuations, both of which can artificially accelerate degradation. ISO 15189 'Medical laboratories Particular requirements for quality and competence' and country specific accreditation schemes require that the laboratory 'develop and document criteria for acceptance or rejection of primary samples'. To achieve this, clinical laboratories need to refer to high quality sources of information (guidelines, recommendations and publications) to help determine their acceptance limits for processing time and temperature extremes for specific analytes.

Materials and methods: The objective of this review is to understand and compare published acceptance limits. The initial focus was on acceptance limits for whole blood stored at room temperature. Stability data and acceptation limits (\%) were reviewed from more than 20 publications (post 2000) by compiling several criteria such as number of patients and statistical analysis methodology. To illustrate this process acceptance limits were compared for three sensitive parameters for chemistry (potassium, phosphate and glucose) and one for endocrinology (PTH).

Results: Significant discrepancies in acceptance limits for potassium and the other parameters were found. Inconsistent statistical analyses and small number of patients enrolled can explain some of these differences. However, they do not explain differences in acceptance limits for potassium that were published (4.8\% vs. $10 \%)$. This could result in a significantly different interpretation of what the maximum allowable transportation time should be for whole blood.

Conclusion: There is a need for better definition of acceptance limits and these limits should be standardised internationally.

*Corresponding author: marc_letondel@europe.bd.com 
P56

\section{Metabolomics technology validated quality markers for biobank plasma samples}

\author{
Kamlage $B^{* 1}$, Schmitz $0^{1}$, Schatz $P^{2}$ \\ ${ }^{1}$ Metanomics GmbH, Berlin, Germany \\ ${ }^{2}$ Metanomics Health GmbH, Berlin, Germany
}

Background: Research in the healthcare area like identification and validation of new diagnostic biomarkers, drug target discovery and treatment monitoring approaches often starts with the analysis of existing biobank samples. The quality of these biobank samples can be impaired by various pre-analytical sample processing steps that will confound the analytical results and decrease the value of research if not identified and addressed properly. Metabolite profiling, also known as "metabolomics", is a well-suited technology to support the identification of technical biomarkers for the quality assessment of biobank samples due to its high sensitivity plus the broad coverage of physiological and chemical processes.

Materials and methods: Human EDTA plasma samples obtained after applying defined pre-analytical confounding factors were subjected to mass-spectrometry based metabolomics including selected targeted platforms $\mathrm{MxP}^{\text {TM }}$ Broad profiling, $M \times P^{\mathrm{TM}}$ Eicosanoids, $M \times \mathrm{P}^{\mathrm{TM}}$ Catecholamines and MxP ${ }^{\mathrm{TM}}$ Lipids.

Results: Metabolomics data sets were analyzed by simple and mixed-effect linear models. Various pre-analytical processes resulted in significant and reproducible changes of the human plasma metabolome. Several metabolites suited as Quality Markers were identified and validated in independent data sets after Bonferroni-Holm correction of the false-positive rate with P-values being $<0.001$.

Conclusions: The plasma metabolome is influenced by the pre-analytical phase. Reproducible and meaningful biomarker research demands standardized protocols for sample handling (qual- ity assurance) as well as a quality control of samples. High-level result interpretation of metabolomics studies requires framework studies to understand the impact of the pre-analytical phase on the results and to elucidate the underlying physiological and chemical mechanisms.

Key words: metabolomics; quality control, pre-analytical variation

*Corresponding author: beate.kamlage@metanomics.de

\section{P57}

\section{MEPS method optimization for forensic toxicological analysis}

\author{
Mayer M1', Romvári Zs' ${ }^{1}$ Porpáczy Z1' Lajtai A², Lakatos Á*2 \\ ${ }^{1}$ University of Pécs Medical School Department of Forensic \\ Medicine, Pécs, Hungary \\ 2University of Pécs Medical School Department of Laboratory \\ Medicine, Pécs, Hungary
}

Background: The more sensitive procedures and instruments allow using smaller sample volumes for toxicological analysis. In addition, strict environmental guidelines, the more expensive chemicals and labor encourage promote the use of micro-techniques. We aimed to optimize the "microextraction by packed sorbent" (MEPS) method for phenylethylamine-type drugs form biological samples.

Materials and methods: We developed a rapid and easy-to-perform micro-extraction method using MEPS to extract stimulants from urine. We compared two reversed phase sorbent (C8 and C18) filled MEPS bin. We optimized the washing and eluting steps to enhance the recovery and to decrease the presence of endogenous substances. After evaporation of the eluate the dry residue was redissolved and analyzed by HPLC-DAD.

Results: The lower limit of detection of drugs of abuse was between 50 and $100 \mathrm{ng} / \mathrm{mL}$ in urine samples. The standard deviation (RSD\%) of the repeatability was acceptable, the recoveries were between $57 \%$ and $92 \%$ for the examined compounds depending on their logP. 
Conclusions: The C18 MEPS bin was found to be effective for sample preparation of drugs of abuse before forensic toxicological analysis. Using this method the time and solvent requirement was significantly reduced compared to our previously used SPE method.

Key words: urine; MEPS; phenylethylamine-type drugs of abuse

*Corresponding author: agnes.lakatos@aok.pte.hu

\section{P58}

\section{The role of clinical signs in successful sweat testing in children}

\author{
Matica J*, Aralica M \\ Clinical Hospital Centre Rijeka, Clinical Department of \\ Laboratory Diagnostics, Rijeka, Croatia
}

Background: According to guidelines for the performance of sweat test for diagnosis of cystic fibrosis, patient's clinical state is important factor for successful collection of sweat after pilocarpine iontophoresis. Aim of the study was to investigate the role of clinical signs such as poor hydration, fasting, elevated body temperature, acute illness, edema and skin lesions on successful sweat collection in children.

Materials and methods: Regarding the quantity of collected sweat participants were divided in two groups: sufficient sweat quantity group (SSQ) and non-sufficient sweat quantity group (NSSQ). There were 117 participants; 90 children (median age 30 (range 1-196) months) in SSQ group and 27 children (median age 25 (range 1-208) months) in NSSQ group. The questionnaire was consisted of five questions regarding clinical state of child (fasting, elevated body temperature, acute illness, edema, skin lesions) and two questions considering poor hydration. The questionnaire was presented to person accompanying tested child (parent or nurse), offering favorable (1 point) and unfavorable (0 points) answers, according to presence or absence of listed clinical signs.
The Fisher's exact test (Medcalc statistical software, Mariakerke, Belgium) was used to calculate the difference between proportions of listed clinical signs in SSQ and NSSQ groups.

Results: Statistical analysis found significant difference between proportions of poor hydrated children in SSQ and NSSQ groups $(P=0.004$ and $P=$ 0.007). This was not the case for fasting, acute illness, elevated body temperature and skin lesions (P-values: $0.309 ; 0.638 ; 1.000$ and 0.661 respectively) between two groups. There was no unfavorable answer related to edema in all tested children.

Conclusion: The study showed the importance of adequate hydration for successful collection of sweat sample, emphasizing crucial role of preanalytical phase.

Key words: sweat test; clinical sign; children; questionnaire; hydration

*Corresponding author: jasminka.matica@ri.t-com.hr

\section{P59}

\section{Verification of claimed limit of detection for the Roche Troponin T hs STAT assay}

Dukic L*, Simundic AM, Malogorski D

Medical School University Hospital Sestre Milosrdnice, Clinical Institute of Chemistry, Zagreb, Croatia

Background: Detection of cardiac biomarker troponin T (cTnT) together with other evidence of myocardial ischaemia makes important criteria for the diagnosis of myocardial infarction (MI). Limit of detection (LoD) is defined as the lowest amount of analyte in a sample that can be detected by given analytical procedure. Establishment of the limit of detection for cTnT has important role in assessment of patient suspected for MI. The aim of our study was to verify the claimed limit of detection of $0.005 \mu \mathrm{g} / \mathrm{L}$ for the cTnT assay (Roche Troponin T hs (high sensitive) STAT (Short Turn Around Time)) according the Clinical and Laboratory Standards Institute (CLSI) EP17-A protocol. 
Materials and methods: Verification of the claimed LoD was performed on Cobas e 411 analyzer (Roche Diagnostics GmbH, Mannheim, Germany). Twenty repeated measurements of pooled plasma samples with concentration of cTnT 0.005 $\mu \mathrm{g} / \mathrm{L}$ were performed.

Results: Claimed LoD for the Roche Troponin Ths STAT assay showed that observed proportion $(20 / 20)$ is in accordance with the expected value (95\%) at concentration of $0.005 \mu \mathrm{g} / \mathrm{L}$.

Conclusions: Our data support the LoD claimed by Roche for Troponin T hs STAT assay.

Keywords: limit of detection; verification; troponin T assay

*Corresponding author: lora.dukic@gmail.com

\section{P60}

\section{New device reduce the pain but increase laboratory variability during diagnostic blood collection}

Lima-Oliveira $\mathrm{G}^{* 1,2}$, Campelo MDR ${ }^{3}$, Valentim $\mathrm{CD}^{3}$, Romano $\mathrm{SJC}^{3}$, Picheth $\mathrm{G}^{2}$, Guidi GC 1,2

\footnotetext{
'Laboratory of Clinical Biochemistry, Department of Life and Reproduction Sciences, University of Verona, Italy

2Post-Graduate Program of Pharmaceutical Sciences,

Department of Medical Pathology Federal University of Parana, Curitiba, Parana, Brazil

${ }^{3}$ Bioanalise Laboratory, Teresina, Piaui, Brazil
}

Background: A new device called Buzzy promotes cold and vibration to relieve venipuncture pain during diagnostic blood specimen collection. The aim of our study was to evaluate the influence of Buzzy ${ }^{\oplus}$ device on routine clinical chemistry testing.

Materials and methods: Blood was collected from 15 volunteers by a single, expert phlebotomist. All patients were maintained seated for 15 minutes to eliminate possible interferences of blood distribution. After that, a vein was located on the left forearm using only a subcutaneous tissue transilluminator device (without tourniquet), and blood samples were collected using a $20-\mathrm{G}$ straight needle (BD Vacuntainer) directly into $5 \mathrm{~mL}$ SST II Advance ${ }^{\circledR}$ vacuum tubes with clot activator and acrylic gel separator. In sequence, an external cold and vibration by Buzzy ${ }^{\circledR}$ was applied on the right forearm for one minute before venipuncture and continued until the end of the same procedure realized in left forearm. The routine clinical biochemistry tests and haemolysis index $(\mathrm{HI})$ were performed in duplicate immediately after centrifugation. The significance of the differences between samples, taking the values from samples collected using only the subcutaneous tissue transilluminator device as the reference ones was assessed by paired Student's t-test after checking for normality.

Results: Statistical significant differences $(P<0.05)$ were observed for: total protein $(P=0.024)$, albumin $(P=0.014)$, urea $(P<0.001)$, creatine kinase $(P$ $=0.004)$, gamma glutamyl transferase $(P=0.023)$, alkaline phosphatise $(P=0.023)$, amylase $(P=$ 0.007), transferrin $(P=0.027)$, total cholesterol $(P<$ 0.001), HDL-cholesterol $(P=0.008)$ and triglycerides $(P=0.010)$. No significant differences $(P>0.05)$ were observed for: glucose $(P=0.694)$, magnesium $(P=0.719)$, phosphate $(P=0.072)$, chloride $(P=$ 0.276), potassium ( $P=0.659)$, sodium ( $P=1.000)$, iron $(P=0.682)$, creatinine $(P=0.776)$, C-reactive protein $(P=0.750)$, uric acid $(P=0.849)$, lipase $(P=$ $0.248)$, lactate dehydrogenase $(P=0.113)$ and $H I(P$ $=0.9885$ ).

Conclusion: The Buzzy ${ }^{\oplus}$ device relieving venipuncture pain during blood specimen collection can be a new source of laboratory variability. It should be used only for laboratory tests that shown none significant differences. Moreover laboratory personnel should train their phlebotomists to verify the laboratory test request before to use Buzzy ${ }^{\circ}$ during blood collection.

Key words: blood collection; phlebotomist; phlebotomy; quality laboratory management; laboratory variability; preanalytical phase, preanalytical variability

*Corresponding author: dr.g.lima.oliveira@gmail.com 
P61

\section{Evaluation of the thrombin tubes and processing by Statspin express for the need of urgent laboratory assays and impact on TAT}

\author{
Meško Brguljan P \\ University Clinic for Respiratory Diseases and Allergy Golnik, \\ Clinical Chemistry Department, Golnik, Slovenia
}

Background: The laboratory, in consultation with the users, shall establish turnaround times (TAT) for each of the examinations that reflect clinical needs. The laboratory shall periodically evaluate whether or not it is meeting the established TATs. In our laboratory, one hour has been defined as TAT for urgent examinations. The preanalytical phase has an important impact on TAT. In order to improve TATs we decided to evaluate the use of Rapid Serum Tube (RST) Plus vacutainer (BD) using StatSpin Express 3 centrifuge for processing (IRIS). The use of RST vacutainers for TDM examinations have not been evaluate till now. Theophylline and digoxin are examination on our urgent repertoire and our aim was to evaluate the use of RST vacutainers and StatSpin processing in the comparison to tubes a traditional centrifugation, used in daily routine.

Materials and methods: 39 samples have collected in parallel to standard gel tubes (SST II, BD) and RST (BD) tubes and allowed to clot for 30 and 5 minutes, respectively. SST II tubes were centrifuged in traditional centrifuge (Rotina 38, Hettich) at $25^{\circ} \mathrm{C}$ for 10 minutes at 3500 RPM and RST tubes for 5 minutes at 5600 RPM in StatiSpin Express 3 centrifuge. The samples were analysed on Cobas 6000 (Roche Diagnostics) with standard procedures. Results were analysed using Medcalc statistical software. TAT (monthly median of urgent requests) was evaluated by LIS (Labis Statistics).

Results: Clinically acceptable performance was demonstrated for both tested parameters, the regression equation for digoxin was $y=0.04296+$ $0.9566 x$ and for theophylline $y=0.6313+1.0082 x$. TATs (time from sampling to report) were reduced from $55 \mathrm{~min}$ to $42 \mathrm{~min}$.
Conclusions: The use of RST tubes and processing in StatSpin centrifuge is appropriate for the whole urgent repertoire at our clinic, including TDM. The change in preanalytical procedure has a great impact on TAT.

Key words: theophylline; digoxin; preanalytical processing; TAT

Corresponding author: pika.mesko@klinika-golnik.si

P62

New vacuum tube for erytrocyte sedimentation rate: a comparison with $K_{2}$ EDTA

Lima-Oliveira G*1,2, Dima F1, Salvagno GL'1, Marcori M', Mainenti $\mathrm{L}^{1}$, Guidi $\mathrm{GC}^{1,2}$

${ }^{1}$ Laboratory of Clinical Biochemistry, Department of Life and
Reproduction Sciences, University of Verona, Italy
2Post-Graduate Program of Pharmaceutical Sciences,
Department of Medical Pathology Federal University of Parana,
Curitiba, Parana, Brazil

Background: The erythrocyte sedimentation rate (ESR) is a nonspecific marker of disease and is often used by clinicians in assisting diagnosis and follow-up of a variety of infectious and inflammatory disorders. Nevertheless, no information is available on the influence of different Vacuum Tubes on ERS analysis. The aim of the present investigation is to compare ESR results obtained on blood specimens collected with two different types of vacuum tubes.

Materials and methods: Blood samples from 20 volunteers were collected by a single, expert phlebotomist. All were maintained seated for $15 \mathrm{~min}$ utes to eliminate possible interferences of blood distribution. After this interval of time, a vein was located on the forearm using only a subcutaneous tissue transilluminator device (without tourniquet), and blood samples were collected using a $20-G$ straight needle (Terumo) directly into 2 different vacuum tubes: Tube I: $2 \mathrm{~mL}$ 4NC ESR Sodium Citrate Premium (Greiner bio-one, GmbH, Krems- 
munster, Austria) and Tube II: $3 \mathrm{~mL}$ Venosafe 5.9 $\mathrm{mg} \mathrm{K}_{2}$ EDTA (Terumo, Europe, Leuven, Belgium). All samples were assayed for ESR on the TEST $1 \mathrm{YDL}^{\circledR}$ (ALIFAX, Padova, Italy). Calibrations were performed according to the instructions provided by the manufacturer. Analytical imprecision, expressed as inter-assay coefficient of variation (CV) and calculated according to internal quality control is $0.8-2.2 \%$. Data were analysed with the paired Student's t-test after checking for normality.

Results: Our results are expressed as mean \pm standard error of the mean (SEM), shown statistically significant difference between Tube I (16 \pm 2 $\mathrm{mm} / \mathrm{h})$ and Tube II (28 $\pm 3 \mathrm{~mm} / \mathrm{h}), \mathrm{P}<0.001$.

Conclusions: This investigation clearly attests that the preanalytical variability might also affect ESR testing, in that the type of the tube can influence test results. Finally, laboratory personnel should validate reference ranges for this new kind of tube before chose to introduce it in routine laboratory.

Key words: blood collection; phlebotomy; quality laboratory management; laboratory variability; preanalytical phase;

*Corresponding author: dr.g.lima.oliveira@gmail.com

\section{P63}

\section{Comparing apples with apples: the need for consistent pre-analytical error classification}

\author{
Glaysher J*, Watson I, Wootton A
}

Department of Clinical Biochemistry, Aintree University Hospitals NHS Foundation Trust, Liverpool, UK

Background: With improvements to analytical quality, the collection and recording of pre-analytical errors is receiving increasing focus. Our laboratory was the first in the UK to enrol in the Australian Key Incident Monitoring and Management Systems QA scheme (KIMMS) which defines a number of pre-analytical error categories. The IFCC Working Group "Laboratory Errors and Patient Safety" has now published a similar list of 16 pre-analytical error categories.

Materials and methods: We designed and implemented a quality system aligned to KIMMS criteria to capture error information directly from the Laboratory Information System. The data are grouped by location and type, before feedback to service users using email of results and face-to-face discussion of their significance.

Results: Since September 2011 our laboratory has recorded an average of 3712 errors/month. In November 2012 the major error was not receiving a required sample type ( $\mathrm{N}=134 ; 2.28 \%$ of total samples received), closely followed by haemolysis (1003; 2.01\%) and collection errors (433; 0.87\%). Some of these data are at variance from KIMMS overall rates, particularly haemolysis (0.3\%).

Conclusions: Despite the apparently straightforward definition of haemolysis, different laboratories categorise results differently. We designate every sample with an un-reportable test, whereas some laboratories only record samples that are completely rejected. Furthermore, the number of samples used as denominator for calculations is also problematic, since some laboratories classify each sample type whilst others count collection events singly. The automated error recording described here is likely to be more efficient than manual counting; significant numbers of errors may go unrecorded with the latter technique.

Consistent recording of errors is required to allow comparison between laboratories. KIMMS and other pre-analytical QA schemes provide a valuable basis for categorisation and collection of laboratory error rates. International agreement is needed to specify inclusion within each error category to ensure comparability of data.

*Corresponding author: jennifer.glaysher@aintree.nhs.uk 
P64

\section{Performance characteristics of a commercially available reagent and dietil-eter for removing lipoproteins}

\author{
Marevic S*, Laskaj R, Sokolic B
}

University Hospital for Infectious Diseases "Dr. Fran Mihaljevic", Department for Medical Biochemistry and Hematology, Zagreb, Croatia

Background: Lipemic samples are a common preanalytical problem and interference of lipids with measurement of routine clinical biochemical tests like enzymes, bilirubin and C-reactive protein (CRP) is significant. We made a short evaluation, of the performance characteristics of a commercially available lipemia clearing reagent and also evaluated the use of dietil-eter for the same purpose, with visibly non-lipemic samples.

Materials and methods: We treated 10 non-lipemic serum samples with a commercially available reagent for removing lipoproteins (LipoClear, StatSpin, Iris, USA). Also 10 other, non-lipemic serum samples were processed with dietil-eter (Kemika, Croatia) for lipid removal. Determination of glucose, urea, creatinine, bilirubin, AST, ALT, GGT, $A L P, L D$ and CRP was done in all samples, before and after removing lipoproteins, on a biochemistry analyzer (Beckman Coulter AU640, USA).

Results: When compared samples before and after treatment with LipoClear (multiplied with 1.2 for dilution correction) we did not found a statistically significant difference for all biochemical tests $(P>0.10)$ except for CRP $(P<0.05)$. In samples before and after removal of lipids with dietil-eter, the unpaired Wilcoxon test for all measured parameters, showed that there was no statistically significant difference $(P>0.10)$.

Conclusions: LipoClear is easy to use and shows good performance characteristics for determination of all our measured parameters except CRP. Dietil-eter is more complicate to use and careful handling of the specimen after centrifugation is necessary because of pipetting the lower layer for analysis. Although we get comparable results for all our parameters and CRP in our non-lipemic samples treated with dietil-eter, one must be careful because we had one CRP result that was not correct, probably due to specimen handling.

Key words: lipemic; LipoClear; dietil-eter

*Corresponding author: sanjamarevic@gmail.com

\section{P65}

\section{Noncognitive factors in preanalytical of CBC measurement: negligible impact or a disastrous mistake?}

Vidranski V, Laškaj R, Kolovrat K,Kozić Dokmanović S, Marević S*, Sokolić B

University Hospital for Infectious Diseases "Dr. Fran Mihaljevic", Department for Medical Biochemistry and Hematology, Zagreb, Croatia

Background: Complete blood count (CBC) is one of the most commonly ordered routine laboratory test. Making great efforts to eliminate cognitive factors still cannot affect those noncognitive ones, whose misinterpretation, if not recognized, may bring the patient into a considerable risk by diagnostic and therapeutic mistakes.

Materials and methods: A total of four patients admitted to our Hospital were enrolled in this case reports. Blood samples were collected with tripotassium EDTA and with sodium citrate respectively. UniCell DxH 800, Beckman-Coulter hematology analyzer was used to perform CBC. Blood smears of samples were made for microscope observation (Olympus BX51). Samples with lipemia were centrifuged on Hettich Universal 320R for $5 \mathrm{~min} / 20^{\circ} \mathrm{C} /$ 4000 RPM. Samples with cold agglutination were heated in waterbath Rowa $\mathrm{GmbH}$ for $10 \mathrm{~min} / 37^{\circ} \mathrm{C}$.

Results: In the first case it was a transient phenomenon-presence of cold agglutination of red blood cells due to a diagnosis of bilateral pneumonia with pleural effusion in the left chest, during antibiotic therapy. The second case discussed the transient EDTA-dependent pseudothrombocyto- 
penia due to antibiotic treatment of erysipelas. The third case was an influence of lipemia on the value of hemoglobin and erythrocyte constants in the individual with previously known hyperlipemia. The fourth case described interference of malaria parasites on results of differential blood cell count.

Conclusions: Misinterpreted results, due above described-noncognitive influences of errors, can give rise to erroneous diagnosis and inappropriate treatment. The present literature about influence of infectious diseases on preanalitical errors is scarce, so these errors should be recognized and described.

Key words: preanalytical; lipemia; pseudothrombocytopenia; cold agglutination; malaria

*Corresponding author: sanjamarevic@gmail.com

\section{P66}

\section{Are patients well informed about proper collection of 24-hour urine sample?}

\author{
Miler $\mathbf{M}^{*}$, Simundic AM \\ University Department of Chemistry, Sestre Milosrdnice \\ University Hospital Center, Zagreb, Croatia
}

Background: Outpatients are usually not quite well informed about the importance of proper urine collection. The proper collection of 24-hour urine sample is important because results of all biochemical parameters depend on its quantity and quality. In this preliminary study we aimed to assess how well outpatients are informed about the policy and procedures for 24-hour urine sample collection in our laboratory.

Materials and methods: Outpatients from Sestre Milosrdnice University Hospital Center with collected 24-hour urine sample were invited to participate in the survey. All patients who have consented to this survey were asked 10 questions about basic demographic data, urine collection, fluid volume intake on the day of urine collection, type of container for collection and collected urine volume. Patients were also asked to state the resource they have used to gather information on how to prepare for urine collection.

Results: We have enrolled 59 outpatients (21 males). Majority of participants were older than 65 years (29/59). Even 20 patients out of 59, did not follow correct urine collection procedure. Almost a quarter of patients either deliberately increases or decreases the fluid volume intake on the collecting day. Although the most (35/59) of the patients answered that the water bottle is the most suitable for urine collection, even 29 participants have chosen used bottle of soft drink, and 3/59 have used the bottle of milk for urine collection. A substantial proportion of patients (4/59) have even discarded a portion of urine that exceeded the bottle volume. When asked to state the resource they have used to gather information, most of the patients (37/59) stated that they were informed by the laboratory staff about the proper urine collection procedure.

Conclusions: A substantial proportion of patients are not following correct procedure for 24-hour urine collection. This clearly indicated the room for improvement. Better education of outpatients is needed. A concerted action of physicians and laboratory staff is the best approach to address this important issue.

Key words: 24-hour urine sample; urine collection; preanalytical factors

*Corresponding author: marijana.miler@gmail.com

\section{P67}

\section{How can we automate the handling of interfering factors in health laboratories?}

Farkas $\boldsymbol{K}^{*}$, Siska A, Valczer E, Telkes M, Seres E

University of Szeged, Dept. of Laboratory Medicine, Szeged, Hungary

Background: There is a wide variety of modern automated systems to handle the effects of interfering factors (e.g. lipaemia, haemolysis etc.) on 
patient's results. Many of them require expansive reorganization of laboratory workflows or new measuring instruments. The aim of this study was to apply these automatisms in our laboratory without changing workflows and instrumentation.

Materials and methods: Automatic index measurements of each serum samples have been introduced. The manual evaluation of serum indexes have been automated by using our Laboratory Information System. Shortly thereafter, a quick, uniform and objective interpretation of interfering factors in our routine laboratory workflow has been realized. In addition, the difference between our previous evaluation strategy and the actually used evaluating protocol was examined between 2007 and 2012.

Results: With this automated method we were able to reduce the errors below $0.005 \%$.

Conclusions: On the basis of our results we could present an almost completely automated solution of handling interfering factors.

Key words: serum index; automation; interpretation; interfering factors

*Corresponding author: lokine.farkas.katalin@med.u-szeged.hu

\section{P68}

\section{Improvement of preanalytical phase quality in laboratories of University Hospital}

Bunešová M*, Moučková Š, Moravcová L

Department of Clinical Biochemistry and Pathobiochemistry, 2nd Medical Faculty, Charles University, University Hospital Motol, Prague, Czech Republic

Objective: The observation of the state and the improvement of preanalytical phase quality by the influence of Society of Clinical Biochemistry Recommendation, accreditation of laboratories and the whole hospital and in the consequences cooperation of laboratories with departments with ambulances and with University Hospital clinics.
Materials and methods: The observation and evaluation of samples that were rejected by laboratories when taking-over due to some preanalytical mistakes. Te observation results were evaluated in relation with the total number of accepted samples as values quality indicators values. The study specially focused on inaccurately identified specimens. The observation was done from 2007 to 2012 at the Institute of Clinical Biochemistry and from 2012 at other Hospital laboratories too).

Results: Since the year 2007 we have been monitoring gradual but permanent growth of preanalytical phase quality. It is manifested by decrease of mistakes, by decrease of rejected samples number, inaccurately identified tubes and by the improvement of numerical values of preanalytical quality indicators identification errors and in the same day repeated requirements. The usage of the hospital information system allows us to differentiate quality of preanalytical phase even according to the level of demands of patients. The number of rejected samples at the emergency $(0.14 \%)$ was compared with the number of rejected samples at the intensive care unit (3.8\%) and with the department of long-term care (2.9\%).

Conclusion: The care of quality improvement of preanalytical phase is secured completely. We use Recommendation of Society of Clinical Biochemistry about the rejection of inappropriate samples and its concordance with the accreditation process according to ISO 15189. The cooperation of laboratories with department managers has been developed, trainings of nurses are being held, the NIS is used, the system of mutual connection between laboratories and departments and in case of need the support of top management is provided even with a possible deployment of deep analysis of serious errors and defects causes. The target is to reduce the risk of patient care in the hospital and for the future to create a pattern that could inspire the whole Czech Republic to improve significantly the preanalytical phase Quality

Key words: defective sample; identification; preanalytical mistakes

*Corresponding author: martina.bunesova@fnmotol.cz 
P69

\section{Pre-analytical factors of routine coagulation assays in Norwegian hospital laboratories}

\author{
Kristoffersen $A^{* 1}{ }^{* 1}$, Stavelin $A^{2}$, Vannes $S^{1}$, Kristensen $G B^{3}$ \\ 'Laboratory of Clinical Biochemistry, Haukeland University \\ Hospital, Bergen, Norway \\ ${ }^{2}$ Norwegian Quality Improvement of Primary Care Laboratories \\ (NOKLUS), Department of Public Health and Primary Health \\ Care, University of Bergen, Bergen, Norway \\ ${ }^{3}$ Norwegian Clinical Chemistry EQA Programme (NKK), Bergen, \\ Norway
}

Background: External quality assessment schemes mainly consider the quality in the analytical phase, but studies have shown that up to $70 \%$ of the errors occur in the pre-analytical phase. Therefore, the Norwegian Clinical Chemistry EQA Programme (NKK) initiated a program on the pre-analytical phase to get an overview on how important aspects in the pre-analytical phase were handled in the hospital laboratories.

Materials and methods: Hospital laboratories in Norway were invited to fill in a web-based questionnaire regarding pre-analytical routines for the most common coagulation assays (APTT, INR, fibrinogen and $D$-dimer). The results focusing on the routines of how to detect and handle high/low hematocrit, hemolysis, bilirubinemia and/or lipemia, are presented.

Results: 57 of 69 (83\%) laboratories responded, of which $\sim 80 \%$ used instruments with mechanical clot detection. Most laboratories (89\%) had no routines for detecting abnormal hematocrit (high/ low). One of the laboratories corrected for low hematocrit, but none corrected for high. After centrifugation, a visual check for hemolysis was performed by $79 \%$ of the laboratories. When hemolysis was detected, $1 / 3$ would reject the sample, $1 / 3$ would report the result with a comment and $1 / 3$ would report the result without a comment. 40\% gave additional comments about sample rejection if strongly hemolysed. Lipemia and bilirubinemia was detected by visual inspection in $57 \%$ and $47 \%$ of the laboratories, respectively. D-dimer results would be reported without a comment despite lipemia or bilirubinemia in $38-63 \%$ of the laboratories answering this question, while this number was $75-91 \%$ for the other analyses. Only one laboratory had an instrument automatically checking for hemolysis, bilirubinemia and lipemia.

Conclusion: There is a large variation in how Norwegian laboratories detect and handle possibly interfering factors when coagulation assays are requested. The guidelines are quite vague on these issues, and it is difficult to assess the clinical consequences of this variation.

Key words: pre-analytical phase; coagulation assays; hemolysis; bilirubinemia; lipemia

*Corresponding author: ann.kristoffersen@helse-bergen.no

\section{P70}

\section{Pre-analytical routines in coagulation testing: are guidelines followed?}

\author{
Kristoffersen $A H^{* 1}$, Stavelin AV2, Vannes $S^{1}$, Kristensen GB3 \\ ${ }^{1}$ Laboratory of Clinical Biochemistry, Haukeland University \\ Hospital, Bergen, Norway \\ ${ }^{2}$ Norwegian Quality Improvement of Primary Care Laboratories \\ (NOKLUS), Department of Public Health and Primary Health \\ Care, University of Bergen, Bergen, Norway \\ ${ }^{3}$ Norwegian Clinical Chemistry EQA Programme, Bergen, \\ Norway
}

Background: It has been documented that about $70 \%$ of errors in laboratory medicine occur in the pre-analytical phase. The aim of this survey was to study pre-analytical conditions of routine haemostasis testing in Norwegian laboratories, and compare them with current guidelines.

Materials and methods: Hospital laboratories in Norway were invited to fill in a web-based questionnaire regarding pre-analytical routines for the routine coagulation assays (APTT, INR, fibrinogen and D-dimer). The part of the questionnaire focusing on the routines for venepuncture (for example needle gauge, type of tube, centrifugation), the accepted filling volume of the tubes and time between blood sampling and analysing (stability), are presented here.

Biochemia Medica 2013;23(1):A1-A55 
Results: 57 of 69 (83\%) laboratories responded. There was good agreement regarding needle gauge, temperature in the centrifuge and type of tube used for sample collection (3.2\% sodium citrate), all (more or less) following the CLSI guidelines (H21-A5). However, large differences in practice were seen amongst the participants regarding for example centrifugation force and time, with a median centrifugation force of $2500 \times \mathrm{g}$ (10 and 90 percentiles $2000-4440 \times$ g) for 10 minutes ( 3 and 15 minutes) and accepted filling volumes of the collection tubes (median 90\% for APTT, fibrinogen and D-dimer and 75\% for INR with 10 and 90 percentiles of $70-100 \%$ for all four analyses). The maximum accepted length of time between blood sampling and analysing the sample (stability) also varied widely, with a median for fibrinogen and Ddimer of 8 hours (10 and 90 percentiles of 1 and 72 hours) and a median of 48 hours for INR (10 and 90 percentiles of 24 and 72 hours), but only 3 hours for APTT (not heparin treated) (10 and 90 percentiles of 1 and 4 hours).

Conclusion: Wide variation was seen in pre-analytical routines in haemostasis testing, often not according to the CLSI guideline.

Key words: pre-analytical phase; coagulation assays; centrifugation; tube fill volume; stability

*Corresponding author: ann.kristoffersen@helse-bergen.no

\section{P71}

\section{Experience in introduction of quality indicators in pre-analytical phase}

\author{
Beletic A*1, Sumarac $Z^{1}$, Milinkovic N1', Ignjatovic S1,2, \\ Majkic-Singh $\mathrm{N}^{1,2}$ \\ ${ }^{1}$ Center for Medical Biochemistry, Clinical Center of Serbia, \\ Belgrade, Serbia \\ 2Department of Medical Biochemistry, Faculty of Pharmacy, \\ University of Belgrade, Serbia
}

Background: The implementation of quality indicators (QI) in pre-analytical phase has not been uniformed yet. Experience in introducing pre-ana- lytic QI in Center for Medical Biochemistry (CMB), Clinical Center of Serbia is presented.

Materials and methods: QI had been introduced in Central Department of CMB, during April 2012. On monthly basis, 31,656 samples were processed: $63.2 \%$ for chemistry, $21.4 \%$ for haematology and $8.8 \%$ for haemostasis tests, while urine and feces samples represented $6.6 \%$. Model of quality indicators-Version 4 issued by IFCC was used as guideline for defining QI.

Results: A total of 18 QI were introduced: 3 for identification (1 for patient and 2 for physician), 4 for test ordering and input and 11 for sample collection, transport and receipt. In $2.42 \%$ of request patient was erroneously identified. Physician's identification was absent in $1.72 \%$ and was not accurate in $2.79 \%$ of requests. Unintelligible requests occurred in $0.58 \%$, while incidences of missing, added and misinterpreted tests were $0.26 \%, 0.02 \%$ and $0.26 \%$ respectively. In certain cases $(0.46 \%)$ sample was not received. QI covering collection of sample was the following: inappropriate container $(0.10 \%)$, clotting $(0.22 \%$ for haematology and $0.00 \%$ for chemistry), insufficient volume $(0.17 \%)$, inadequate sample-anticoagulant volume ratio $(0.27 \%)$. No samples were damaged in transport, while errors occurred in labeling (0.09\%) and storage $(0.03 \%)$. Haemolysis was present in samples for haemostasis $(0.12 \%)$ and chemistry testing (0.98\%).

Conclusion: The abovementioned QI provide enough confidence for evaluating pre-analytical phase in $\mathrm{CMB}$ and indicate steps which require improvement. This positive pilot experience strongly points towards the need for their further implementation and development through the activities governed by IFCC Working Group on laboratory errors and patient safety.

Key words: quality indicators; pre-analytical phase

*Corresponding author: andjelo.beletic@yahoo.com 
P72

\section{Unsuitable specimens in clinical practice: before and after an educational lecture}

\author{
Čeprnja M*, Serdar T, Hodak N, Živković M, Marijančević D, \\ Romić Ž \\ Clinical Department for Laboratory Diagnostic, University \\ Hospital Dubrava, Zagreb, Croatia
}

Background: Analyzing inadequate samples or incorrectly identified samples can lead to fatal consequences, so it is extremely important not to accept them for laboratory testing. Egregious errors of this type can be prevented by educating medical personnel on sampling and handling of the sample prior to measurement and educating laboratory staff thereof and further about acceptance of samples.

The aim of this study was to determine whether an educational lecture had any effect on the quantity of the inadequate samples submitted to the Clinical Department of Laboratory Diagnostics, University Hospital Dubrava.

Materials and methods: The collected data were divided into two groups. The first group contains data on all samples received during the 60 days prior to the training classes held, and the other group of samples during the 60 days after the lecture. The lecture was based on the instructions on the collection of primary samples for medical-biochemical laboratory and was followed by presentations of most frequent preanalytical errors and causes that lead to them. All statistical calculations were performed using Chi sqare test, MedCalc 9.2.0.0. software (Mariakerke, Belgium).

Results: No statistically significant difference was found for hemolysed sera samples (Chi square = $0.113, P=0.737$ ), hemolysed coagulation samples (Chi square $=0.798, P=0.3717$ ), for clot in whole blood and coagulation plasma samples (Chi square $=2.293, P=0.13$ ) in the number of received inadequate samples before and after organized educational lectures.
Conclusion: Regardless of the educational presentation we found no significant decrease of inadequate samples submitted in Clinical Department for Laboratory Diagnostic. However, it is necessary to take into consideration the sampling of "difficult patients" that makes certain percentage of inadequate samples inevitable.

Key words: education lecture; unsuitable specimens; hemolysis

*Corresponding author: marinaceprnja@gmail.com

\section{P73}

\section{Risk analysis for non-analytical EQA in Australasian KIMMS program}

\author{
Petinos P*, Sikaris KA \\ RCPAQAP KIMMS Program, Sydney, Australia
}

Background: There are many key indicators of pathology quality that lie outside the analytical phase. These should ideally be monitored and compared against benchmarks achieved by similar laboratories in order to assure performance standards are being met. These incidents vary in type from the reliable labelling of patients samples including the identification of samples mislabelled with another patient's name to the receipt of samples which are received haemolysed and unable to be used for the analysis of some assays. The ability of the laboratory to detect these errors may vary according to the incident type and also vary in the clinical impact on the patient from the inconvenience in collecting another sample to the potentially extreme consequences of transfusing with the wrong blood type.

Materials and methods: We have graded these incidents according to the ability to detect the error and its consequence together with the frequency of that error using the tool of 'Failure Mode and Effect Analysis' (FMEA) to create a risk score for each of the incident types in our EQA program. A risk matrix is reported as part the EQA report to participants which allows the quality managers to 
focus their attention on the incidents with the greatest overall risk in their laboratory.

Results: Examples of incidents that have high risk scores are (i) Report retraction incidents: low frequency errors that could result in unnecessary or delayed patient management (ii) Haemolysed sample incidents: judged as a high risk category due to the large frequency rather than any difficulty in detecting or typically minor consequence (iii) Transfusion sample identification incidents: 'nearmiss' errors with potentially life threatening consequences.

Conclusion: While in the ideal world all these quality incidents should be addressed, in the real world we practically should start with the incidents judged to have the greatest overall risk to patient care.

Key words: EQA; risk analysis; error detection; risk matrix

*Corresponding author: Penny.Petinos@rcpaqap.com.au

\section{P74}

\section{Improvements in the admission of outpatients at the Department of Laboratory Diagnostics, University Hospital Center Zagreb}

\author{
Fuček M*, Alpeza Viman I, Zekušić M, Herceg A, Sertić J \\ Department of Laboratory Diagnostics, University Hospital \\ Centre Zagreb, Croatia
}

Background: On average, about 200 outpatients are admitted daily at the Department of Laboratory Diagnostics (DOLD), University Hospital Center Zagreb. Relocation of the Clinical Unit for Patient Admission and Management to new premises has entailed reorganization of outpatient admission and sampling of biological material. HAVIS, an audio-visual system for calling out patients has been integrated with the currently used hospital (HIS) and laboratory information system (LIS), while blood sampling test-tubes are barcode labelled in advance.
Materials and methods: Upon arrival to laboratory reception desk, patients present the referral for laboratory tests and are given a number generated by the HAVIS system. A copy of the number is enclosed with the referral, and basic patient data and laboratory tests are entered into HIS. All test requests are transferred to LIS using the HL7 communication protocol.

Phlebotomy is performed in five separate rooms in order to ensure patient privacy. A computer is in each of the rooms, with all three applications (HIS, LIS and HAVIS). A patient is referred to the room for venipunction through the HAVIS system, i.e. digital voice and visual information on a display in the laboratory waiting room. After checking patient's identity, laboratory staff performs blood sampling. Information on laboratory tests for a selected patient is displayed on computer screen at the LIS interface entitled: "Entered requests", and data on a number and type of previously labelled test-tubes are added. After sampling, samples are transported in closed containers to the worksite.

Results: In the first half of year 2012, 22,926 outpatients were managed at DOLD. Average time from presentation of referral to patient's entry into phlebotomy room was 0:35:18 minutes (min: 0:14:14, max: 0:58:33), depending on the type and complexity of referral. Application of test-tubes labelled in advance reduced patient management time and ensured privacy (patient's name and surname are not written on the test-tube). Also, expansion of the existing LIS with a module that, depending on the number of requested tests, determines the minimum number of necessary blood test-tubes, helped reduce the number of unnecessarily sampled test-tubes.

Conclusion: Better work organization and implementation of information systems improved the entire process of laboratory management of outpatients and thus contributed to increased quality of patient health care.

Key words: audio-visual system for calling; testtubes labelled in advance

*Corresponding author: mfucek@kbc-zagreb.hr 
P75

\section{Verification of lipemia interference for routine clinical chemistry}

\author{
Nikolac N*, Miksa M, Saracevic A, Simundic AM
}

University Department of Chemistry, Medical School University Hospital Sestre Milosrdnice, Zagreb, Croatia

Background: Lipemia interferes with large number of laboratory tests. In order to assess the extent of lipemia, each manufacturer of laboratory reagents should provide data on interference as part of analytical specification of the test. Laboratory customers should verify those recommendations in laboratory setting. Aim of this study was to verify manufacturer's specifications regarding lipemia for Beckman Coulter clinical chemistry parameters. Also, since allowable bias by manufacturer was arbitrary $(3,5$ or $10 \%)$, we aimed to compare our results with desirable specifications for imprecision derived from intra- and inter-individual biologic variation.

Materials and methods: In 20 different serum pools following parameters were determined: total and direct bilirubin, urea, creatinine, glucose, calcium, AST, ALT, CK, CK-MB, LD, ALP, GGT, amylase, lipase, total proteins, CRP, phosphates, magnesium, iron, albumin, sodium, potassium and chlorides. All measurements were performed on Beckman Coulter AU680 analyzer (Tokio, Japan) using original reagents and applications as recommended by manufacturer (Beckman Coulter, Inc., Brea, CA, USA). Samples were then spiked with triglyceride rich infusion of $20 \%$-Intralipid ${ }^{\circledR}$ (Fresenius Kabi $A B$, Uppsala, Sweden) in series of concentrations corresponding to cut-off values declared by manufacturer (100, 300, 500, 700, 800, 900 and 1000 mg/dL Intralipid ${ }^{\circledR}$ ) and analytes were determined in each sample. Bias was calculated for each concentration and compared to declared specification.

Results: Out of 24 tested parameters, manufacturers recommendations were confirmed for 12 (lipase, direct bilirubin, urea, calcium, total proteins, CRP, iron, GGT, albumin, sodium, potassium, chlo- rides). However, declared bias of lipemic samples

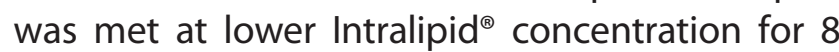
analytes (bilirubin, AST, CK, CK-MB, LD, amylase, magnesium and ALP) suggesting stronger influence of lipemia than declared by manufacturer. Also, for 4 analytes (creatinine, glucose, ALT, phosphates) we have observed higher acceptable cutoff value than specified. The most significant deviation was observed for CK, magnesium and amylase (manufacturers cut-off vs. laboratory cut off: 1000 vs. 300 mg/dL Intralipid ${ }^{\circledR}$ ).

Conclusions: Our results indicate significant differences in lipemia interference compared to manufacturer's recommendations for Beckman Coulter clinical chemistry parameters.

Key words: interference; lipemia; analytical specifications

*Corresponding author: nora.nikolac@gmail.com

\section{P76}

\section{Are differences in PT, aPTT and fibrinogen between the first and the second blood draw significant?}

\author{
Celap I*, Simundic AM, MargetićS
}

Clinical Institute of Chemistry, University Hospital Center Sestre milosrdnice, Zagreb, Croatia

Background: Preanalytical phase in plasma-based haemostasis testing is source of numerous errors. Among others, sample collection represents one of the most important elements of preanalytical phase in coagulation testing. Tourniquet time, test tube filling and mixing as well as the order of draw are recognised as fundamental factors which have influence on the haemostasis results. CLSI H3-A6 and CLSI H21-A5 guidelines stated that is not necessary to discard the first tube if only coagulation tests are requested. However, this refers only for routine blood coagulation assays (PT, aPTT). Nevertheless, statistically, though not clinically, significant differences for routine blood coagulation tests results have been demonstrated in a large 
patient group. The aim of this work was to examine the differences in routine coagulation tests results between the first and the second blood tube.

Materials and methods: For 171 subjects, blood was drawn in the first and the second evacuated tube containing sodium citrate (3.2\%). Paired samples were analysed for PT, aPTT and fibrinogen at the same time on the coagulation analyser BCS (Siemens, Marburg, Germany). The tests were performed using the original reagents. Bias was calculated between the first and second draw and compared with desirable imprecision based on biological variation $(0.5 \mathrm{CVw})$. Analytical CVs were $4.5 \%$, $3.6 \%$ and $4.5 \%$ for PT, aPTT and fibrinogen, respectively.

Results: The difference between the first and the second draw was not acceptable for 96/171 (56\%) of patients for PT, in 32/171 (19\%) patients for aPTT and in 27/171 (16\%) patients for fibrinogen. Differences for PT, aPTT and fibrinogen were outside the limits of analytical imprecision in our laboratory in $16 \%, 13 \%$ and $22 \%$ patients, respectively.

Conclusion: Differences in PT, aPTT and fibrinogen level between the first and the second blood draw exceed the limits of desirable imprecision based on biological variation.

Key words: coagulation testing; biological variation; discard tube

*Corresponding author: ivana.celap@gmail.com

\section{P77}

\section{Evaluation of high speed centrifugation and Lipoclear ${ }^{\circledR}$ reagent for lipemia removal}

\section{Saracevic A*, Nikolac N, Simundic AM}

University Department of Chemistry, Medical School University Hospital Sestre Milosrdnice, Zagreb, Croatia

Background: Lipemic samples are a common problem in everyday laboratory practice; however there are not many studies that have evaluated protocols for lipemia removal. The aim of this study was to evaluate the test recovery after lipid removal with high speed centrifugation and after processing the samples with commercially available Lipoclear ${ }^{\circledR}$ reagent.

Materials and methods: Plasma pool was collected for the purpose this study. The samples were spiked with fat emulsion Intralipid ${ }^{\circledR}$ (Fresenius Kabi $A B$, Uppsala, Sweden) to create final concentrations of 300 and $500 \mathrm{mg} / \mathrm{dL}$ Intralipid ${ }^{\circledR}$. Aliquotes of these lipemic samples were centrifuged at $12,100 \mathrm{~g}$ for 5 minutes or processed with Lipoclear ${ }^{\circledR}$ reagent (Iris International, Inc., Westwood, MA, USA) according to the original manufacturer's protocol. The following biochemical parameters were determined in native plasma, lipemic plasma and in samples after lipemia removal: bilirubin, direct bilirubin, urea, creatinine, glucose, sodium, potassium, chlorides, calcium, phosphates, magnesium, iron, AST, ALT, CK, CK-MB, LD, ALP, GGT, amylase, lipase, total protein, albumin, CRP, troponin $T$ and proclacitonin. All measurements were performed in triplicates on Beckman Coulter AU680 analyzer (Tokio, Japan) using original reagents (Beckman Coulter, Inc., Brea, CA, USA), except for troponin T and procalcitonon which were measured on Cobas E411 analyzer (Roche Diagnostic, Mannheim, Germany) with the original Roche reagents. The recovery for each parameter after Lipoclear $^{\circledR}$ and centrifugation was calculated and compared with desirable specification for imprecision (DSI) according to Ricos et al. (except for procalcitonin according to Barassi).

Results: DSI criteria for recovery were not met for calcium, total protein, sodium and chlorides after high speed centrifugation and for glucose, calcium, phosphates, magnesium, sodium, potassium, chlorides, ALP, GGT, CK-MB, total protein, albumin and troponin $T$ after using Lipoclear ${ }^{\circledR}$. Overall, the lowest recovery was found for CRP (6.27\%) after the use of Lipoclear ${ }^{\circledast}$.

Conclusions: As of our results, high speed centrifugation of lipemic samples is a more acceptable method for lipemia removal than the commercially available Lipoclear ${ }^{\circledR}$ reagent.

Key words: interference; lipemia; test recovery

*Corresponding author: andrea.saracevic@gmail.com 
P78

\section{Teaching preanalytics in biomedical laboratory science}

\section{Penttinen U}

Biomedical Laboratory Science, Novia University of Applied Sciences, Vaasa, Finland

In Finland education in biomedical laboratory science (BLS) is given by Universities of Applied Sciences (UAS) in Helsinki (Helsinki Metropolia UAS), Turku (Turku UAS), Kuopio (Savonia UAS), Tampere (Tampere UAS), Oulu (Oulu UAS), and Vaasa (Novia UAS). In Novia, teaching language is Swedish, in the rest Finnish. The education takes 3.5 years, and comprises 210 ECTS credits. Laboratory practice is included in the studies $(75 \mathrm{cr})$. The students make a thesis of $15 \mathrm{cr}$. The biomedical laboratory scientist is a bachelor of health care. The curriculum for BLS is not strictly regulated by the government. However, the education differs only slightly, because all six universities have agreed on many issues during their yearly network meetings.

In 2010 Novia UAS took preanalytics as one of the leading themes for BLS. Preanalytical factors should be considered in almost every course. During their education students collect information on preanalytics from different courses and their practice to make a synopsis. Most of the teaching in preanalytics is about collecting specimens. This is the main subject in the course "Basics in Bioanalytics" (6 cr). Specimen collection is considered for bioanalysis in hematology, biochemistry, microbiology, histopathology, cytology, genetics, immunology, and transfusion medicine. Patient contact and supervision have a great impact on the preanalytical phase. Students also have a clinical practice in specimen collection $(3 \mathrm{cr})$.

The preanalytical phase takes a small part of most courses, in credits it is approximately 10 . The preanalytical phase is given about $19 \mathrm{cr}$ in the education programme for BLS. Depending on the thesis subject the preanalytical phase is even more represented. Novia UAS is going to have a project on preanalytics together with the laboratories of Vaa- sa Central Hospital. Students can make their thesis in this project.

Key words: the preanalytical phase; teaching preanalytics; biomedical laboratory science (BLS) education in Finland; Novia University of Applied Sciences; basics in bioanalytics

Corresponding author: ulla.penttinen@novia.fi

\section{P79}

\section{Preanalytical stability of serum Anti-Mullerian hormone (AMH) and follicle stimulating hormone (FSH) in different conditions}

\section{Hepnar D}

Sang Lab - klinická laboratoř, Karlovy Vary, Czech Republic

Background: Serum Anti-Mullerian hormone $(\mathrm{AMH})$ and follicle stimulating hormone (FSH) levels are most used markers for evaluation of infertility and to control response by ovarian hyperstimulation. The aim of the study is to verify preanalytical stability under different conditions.

Materials and methods: Preanalytical stability was monitored for 50 human serum samples for the commonly used conditions of storage before analysis in the laboratory $\left(+20^{\circ} \mathrm{C},+4{ }^{\circ} \mathrm{C}\right)$. Samples were measured 2 hours after blood collection, then 5 days after first measurement. Tests were measured on Roche Modular system, $\mathrm{AMH}$ on AMH Gen II ELISA Beckman Coulter diagnostics kit. Percentages differences and paired test were used for statistical evaluation (Statistica, StatSoft).

Results: At a temperature of $20^{\circ} \mathrm{C}$ from the initial values of AMH (0.35 to $11.90 \mathrm{ng} / \mathrm{mL}$ ) increased on average by $32 \%$ (from 0.42 to $15.61 \mathrm{ng} / \mathrm{mL}, 95 \% \mathrm{Cl}$ ), from baseline FSH (2.0 to $62.6 \mathrm{lU} / \mathrm{L}$ ) increased by an average of $8.7 \%$ (2.3 to $69.2 \mathrm{IU} / \mathrm{L}, 95 \% \mathrm{Cl}$ ). At 4 ${ }^{\circ} \mathrm{C} \mathrm{AMH}$ values increased on average by $27.2 \%$ (from 0.41 to $16.22 \mathrm{ng} / \mathrm{mL}, 95 \% \mathrm{Cl}$ ), FSH values increased on average by $5.1 \%(2.2-64,4 \mathrm{IU} / \mathrm{L}, 95 \% \mathrm{Cl})$.

Conclusion: $\mathrm{AMH}$ and $\mathrm{FSH}$ at the temperature +20 ${ }^{\circ} \mathrm{C},+4{ }^{\circ} \mathrm{C}$ after 5 days of storage is unstable and is 
necessary to find another way of storage. Storage system under test is not suitable for evaluation of infertility and to control response by ovarian hyperstimulation.

Key words: $\mathrm{AMH}$; $\mathrm{FSH}$; storage; stability

Corresponding author: david.hepnar@sanglab.cz

\section{P80}

\section{Implementation of "A combined electronic requests system for diagnostic tests and appointments"}

\author{
Guaita $M^{*}$, de Miguel A, Marcaida G,Miguel A, Doñate \\ V,Palomino JF, García A \\ Centro de Diagnóstico Biomédico (CDB), Hospital General \\ Universitario de Valencia, Valencia, Spain
}

Background: One of the main objectives of CDB is to incorporate ICTs to improve the quality in attending to the patients. For this reason, the combined electronic requests system for diagnostic tests and appointments is designed and implemented for specimen collections and medical consultations with the following objectives: 1) To facilitate the patient appointment process; 2 ) To develop an easy and friendly electronic request system; 3) To simplify the patient appointments; 4) To have a tool to modulate the request; 5) To improve the quality of specimen collection.

Materials and methods: 1) Composition of work groups for the design of a request form format; 2) Design of the request form and the development of the summons in collaboration with Connect All Systems; 3) Pilot trial with a group of interdisciplinary faculties; 4) Progressive incorporation programmed for consultations.

Results: The goals achieved are: easy to use integrated analytical requests; interactive e-form that allows the dynamic request of additional information for determined parameters; development of a tool that allows the request of multiple specimens in the same e-form; improvement in the quality of administrative and clinical information: correct identification of the patient, as well as of the requester and their specialty and availability for clinic orientation for $100 \%$ of the patients; time reduction in the administrative process for patients; improvement in the circuits and ease of access by redirecting the patient to his primary care centre for specimen collection; improvement of the pre-analytical phase by giving written information at the appointment, detailing the preparation required.

Conclusions: A combined system of e-requests and appointments has been achieved and adapted to the clinic's needs in a progressive and organized way. This innovation has been well received and the clinics have felt part of the entire process.

*Corresponding author: guaita_marmar@gva.es

\section{P81}

\section{Impact of pre-analytical parameters on the measurement of circulating microparticles}

Judicone $C^{* 1}$, Lacroix R ${ }^{2,3}$, Poncelet P1, Mooberry M4, Key NS 4 , Dignat-George F2,3

\footnotetext{
${ }^{1}$ BioCytex Company, Marseille, France

${ }^{2}$ Aix Marseille Université, INSERM, VRCM UMR_S1076, UFR de Pharmacie, Marseille, France,

${ }^{3} \mathrm{APHM}$, Hôpital de la Conception, Laboratoire d'Hematologie et de Biologie Vasculaire, Marseille, France,

${ }^{4}$ Department of Medicine, University of North Carolina, Chapel Hill, NC, USA
}

Background: Microparticles (MP) are small vesicles of 0.1-1 $\mu \mathrm{m}$ released in response to activation or apoptosis, considered as interesting biomarkers and biovectors in coagulation, inflammation and cancer. Many clinical studies have already tried to assess their potential for identification of patients at cardiovascular risk but pre-analytical steps remain an important source of variability and artifacts. We aimed at assessing the impact of various pre-analytical parameters on MP measurement, including type of collection tube, phlebotomy conditions, transportation practices, centrifugation steps and freezing. 
Material and methods: One tube from each of at least 10 healthy volunteers was used for each tested condition. MP were assessed by three methods: flow cytometry, a thrombin generation test (Calibrated Automated Thrombogram ${ }^{\oplus}$ ) and a phospholipid-dependent clotting time (STA ${ }^{\circledR}$-ProcoagPPL, Stago).

Results: Our data indicated that: 1) Citrate provides the lowest MP counts among all tested anticoagulants (CTAD $+20 \%$, Heparin $+370 \%$, EDTA $+1020 \%$ ). 2) A time delay before first centrifugation increases PMP counts $(+40 \% \pm 35 \%, P=0.02)$ and activity as soon as 1h. 3) Agitation is critical for PMP analysis $(+10 \%$ for gentle agitation vs. $+250 \%$, $\mathrm{P}<$ 0.01 for strong agitation). This effect can be prevented using a well controlled transportation system. 4) A routine-oriented centrifugation protocol $(2 \times 2,500 \times \mathrm{g} 15 \mathrm{~min})$ generates less artifactual PMP counts than the previous ISTH-recommended protocol $(1,500 \times \mathrm{g} 15 \mathrm{~min}+13,000 \times \mathrm{g} 2 \mathrm{~min})$ $(+170 \% \pm 120 \%, P<0.001) .5)$ One freezing/snap thawing cycle at $-80{ }^{\circ} \mathrm{C}$ does not significantly alter PMP counts and procoagulant potential even after 6 months of storage.

Conclusion: Based on these data, a new pre-analytical protocol has been proposed and challenged in a multicentre workshop including 15 laboratories. This study showed a reduction of the interlaboratory variability in both PMP counts when the common protocol was compared with homemade protocols. Standardizing the pre-analytics prior to MP analysis is a mandatory step for any relevant evaluation of these potential biomarkers in clinical studies.

*Corresponding author: coralie.judicone@biocytex.fr
P82

\section{Interference verification of three creatinine methods}

Leonetti R, Curtin C, Sharman $\mathbf{H}^{*}$, Horvath AR

SEALS Department of Clinical Chemistry, Prince of Wales Hospital, Sydney, Australia

Background: Interferences with creatinine measurements may result in inaccurate estimations of the glomerular filtration rate, particularly in children and neonates. We investigated the impact of common interferences on three creatinine methods.

Materials and methods: Pooled serum samples at three creatinine concentrations representative of different age groups were spiked with $\mathrm{HbA}, \mathrm{HbF}$, unconjugated bilirubin, ditaurobilirubin and Intralipid to mimic common interferences, and measured in duplicates with Roche's compensated, rate-blanked, kinetic Jaffe and enzymatic creatinine methods and on Radiometer's ABL827 analyser. Absolute and relative differences between spiked and unspiked specimens were compared to interference indices recommended by manufacturers. Our interference criterion was $\pm 8 \%$ recovery of unspiked samples, based on the allowable limits of performance set by the external quality assurance program of the Royal College of Pathologists of Australasia.

Results: The degree of $\mathrm{HbA}$ and $\mathrm{HbF}$ interference in the Roche Jaffe and enzymatic methods depended on creatinine concentration. The manufacturer's single $\mathrm{HbA}$ haemolysis index (1000 Jaffe; 800 - enzymatic) was only applicable when creatinine was $>100 \mu \mathrm{mol} / \mathrm{L}$. When creatinine was $<100 \mu \mathrm{mol} / \mathrm{L}$, as commonly seen in neonates and children, the interference criterion was met at much lower indices (200 - Jaffe; 500 - enzymatic). $\mathrm{HbF}$ interference makes the Jaffe test unsuitable in neonatal specimens and the enzymatic method, up to $\mathrm{HbF}<6 \mathrm{~g} / \mathrm{L}$, is recommended by the manufacturer. However, in neonates the enzymatic assay already underestimated creatinine by $20 \%$ when $\mathrm{HbF}$ was 3-4 g/L. Unconjugated bilirubin did 
not interfere up to $700 \mu \mathrm{mol} / \mathrm{L}$ (Jaffe) and 300 $\mu \mathrm{mol} / \mathrm{L}$ (enzymatic; manufacturer's recommendation: 171 and 342, respectively). Conjugated bilirubin interfered up to $50 \mu \mathrm{mol} / \mathrm{L}$ (Jaffe) and 200 $\mu \mathrm{mol} / \mathrm{L}$ (enzymatic; manufacturer's recommendation: 86 and 257, respectively). However, the Roche instrumentation cannot differentiate between unconjugated and conjugated bilirubin when index measurements are taken, and therefore the smallest common denominator can only be used by the laboratory's autovalidation system, i.e. $50 \mu \mathrm{mol} / \mathrm{L}$ (Jaffe) and $200 \mu \mathrm{mol} / \mathrm{L}$ (enzymatic) for all types of icterus, unless reflex testing algorithms are introduced at various icterus index thresholds which trigger the measurement of direct bilirubin and the reassessment of the validity and manual validation of results by skilled laboratory staff. The Radiometer ABL 827 creatinine method was not affected by any of the above interferences.

Conclusion: Creatinine method interference by haemolysis and bilirubin can be additive, therefore we found the Radiometer ABL 827 enzymatic method the most reliable for such specimens. Interference indices provided by manufacturers should be critically evaluated at different analyte concentrations, and single cut off values cannot be universally applied to all specimens without verification. Interference acceptance criteria should be agreed internationally and should be based on analytical, biological and clinical impact considerations to guide manufacturers in setting interference indices or cut-offs for various biomarkers.

*Corresponding author: heather.sharman@sesiahs.health.nsw. gov.au
P83

\section{Validation of different specimen types and common interference indices for the Roche high sensitivity cardiac Troponin T assay}

\author{
Leonetti R', Curtin C', Sharman $\mathbf{H}^{* 1}$, Varndell W' ${ }^{2}$, Horvath $\mathrm{R}^{1}$ \\ 1SEALS Department of Clinical Chemistry, Prince of Wales \\ Hospital, Sydney, Australia \\ 2Department of Emergency, Prince of Wales Hospital, Sydney, \\ Australia;
}

Background: Laboratories face increasing demands to improve turnaround time (TAT), particularly in the context of urgent troponin testing in patients presenting with acute chest pain. Poor sample quality is often an obstacle in achieving fast TATs. Plasma specimens are preferred when TAT is critical for testing, however, its use has potential disadvantages: 1) results for some tests may differ due to different sample matrix, necessitating different reference intervals if serum specimens are retained for 'non-urgent' testing; 2) stability of some analytes may be reduced in plasma gel tubes; 3) a small percentage of plasma samples may exhibit falsely elevated troponin results due to fibrin strands, platelets and other cellular material. Hemolysis has been reported to be $4-8.8 \%$ for samples collected in emergency departments and the Roche hs-TnT assay is affected by haemolysis. In the BD Vacutainer ${ }^{\circledR}$ Rapid Serum Tube (RST) thrombin is added to fasten clotting and serum specimens can be obtained within a few minutes. Therefore we compared the hs-cTnT assay (Roche) on specimens collected in BD Vacutainer ${ }^{\circledR} \mathrm{SST}^{\mathrm{TM}} \|$ Advance tubes (SST ${ }^{\mathrm{TM} I \mathrm{I})}$ with those obtained using RST tubes. We also verified the interference indices provided by the hs-cTnT test manufacturer.

Materials and methods: Twenty eight pairs of tubes (SST ${ }^{\mathrm{TM}}$ II and RST) were collected in random order from adults presenting to the Emergency Department with angina. Hs-TnT was measured on Cobas e601 (Roche) and stability of cTnT over a seven day period was determined in both types of samples. The Haemolysis Index (HI) determined by 
the Roche instrumentation for each specimen was also recorded. All analytical comparison data were analyzed by Passing-Bablock regression and difference plots in two groups, i.e. all observations regardless of the $\mathrm{HI}$ value and where the $\mathrm{HI}$ was less than 65 . For the interference index verification study various concentrations of hemoglobin, bilirubin, or Intralipid ${ }^{\circledR}$ were added into $\mathrm{SST}^{\mathrm{TM}}$ ॥| serum with 3 different concentrations of TnT to simulate haemolytic, icteric, or lipaemic samples.

Results: Lower HI was observed in the RST specimens (mean: 22) compared to the SST ${ }^{\mathrm{TM}}$ II tubes (mean: 46; $\left.y=0.1617 x+14.9, r^{2}=0.37\right)$. Hs-cTnT results in both tube types showed a good correlation in all specimens $\left(y=1.0285 x-0.125, r^{2}=\right.$ $0.9964)$ and especially in the tubes with a $\mathrm{HI}<65$ (1.022x $\left.-0.2288, r^{2}=0.9965\right)$. The mean difference of hs-cTnT was $-2.7 \%$ in RST and $+1.2 \%$ in SST ${ }^{\text {TM }}$ II after 24 hours at $25^{\circ} \mathrm{C}$ and both tubes preserved the baseline Troponin concentrations up to 3 days when stored at $4{ }^{\circ} \mathrm{C}$.
Haemoglobin $\mathrm{A}$ at $1 \mathrm{~g} / \mathrm{L}$ (i.e. at $\mathrm{HI}=100$ recommended by Roche), did not affect hs-cTnT results. At $2 \mathrm{~g} / \mathrm{L}(\mathrm{HI}=200)$ there is an underestimation of of hs-cTnT of about $5 \%$ which is clinically acceptable. The magnitude of these interference patterns was the same across various TnT concentrations. Unconjugated bilirubin up to $700 \mathrm{umol} / \mathrm{L}$ and conjugated bilirubin up to $1000 \mathrm{umol} / \mathrm{L}$ had little effect on the hs-cTnT estimation (Roche index: 462). The Lipaemia Index of 2250 by Roche was confirmed in our study.

Conclusions: RST specimens yield concordant hscTnT results with SST ${ }^{\mathrm{TM}}$ II specimens using the Roche hs-cTnT assay. RST tubes produce a "cleaner" serum specimen within max. 10 minutes and are less prone to haemolysis than the $\mathrm{SST}^{\mathrm{TM}} \|$ tubes. The RST tubes seem to be a better alternative for urgent hs-cTnT testing using the Roche assay. The Roche interference indices are widened for haemolysis and icterus which allows the release of critical test results in a larger proportion of patients needing acute assessment and care.

*Corresponding author: heather.sharman@sesiahs.health. nsw.gov.au 